\title{
INDECOMPOSABLE REPRESENTATIONS OF SEMISIMPLE LIE GROUPS
}

BY

BIRGIT SPEH ${ }^{1}$

\begin{abstract}
Let $G$ be a semisimple connected linear Lie group, $\pi_{1}$ a finite-dimensional irreducible representation of $G, \pi_{2}$ an infinite-dimensional irreducible representation of $G$ which has a nontrivial extension with $\pi_{1}$. We study the category of all Harish-Chandra modules whose composition factors are equivalent to $\pi_{1}$ and $\pi_{2}$.
\end{abstract}

Introduction. In a series of articles [5]-[8], I. M. Gelfand, Graev and Ponomarev classify all indecomposable Harish-Chandra modules of $\mathrm{Sl}(2, C)$. Since two representations with different infinitesimal characters cannot both be composition factors of the same indecomposable Harish-Chandra module, it suffices to classify the category $H(\lambda)$ of indecomposable Harish-Chandra modules with a given infinitesimal character $\lambda$. I. M. Gelfand, Graev and Ponomarev show that for every infinitesimal character of $\mathrm{Sl}(2, \mathrm{C}) H(\lambda)$ is equivalent to a subcategory of a category which can be described as follows. The objects of $\mathfrak{X}$ are pairs $V_{1}, V_{2}$ of finite-dimensional complex vector spaces together with linear maps

$$
d^{+}: V_{1} \rightarrow V_{2}, \quad d^{-}: V_{2} \rightarrow V_{1}, \quad \delta: V_{2} \rightarrow V_{2}
$$

with the property that

(a) $d^{+} d^{-}, d^{-} d^{+}$are nilpotent,

(b) $\delta$ is nilpotent,

(c) $\delta d^{+}=d^{-} \delta=0$.

The morphisms are all pairs $\left(\gamma_{1}, \gamma_{2}\right)$ of linear maps such that the following diagram is commutative:

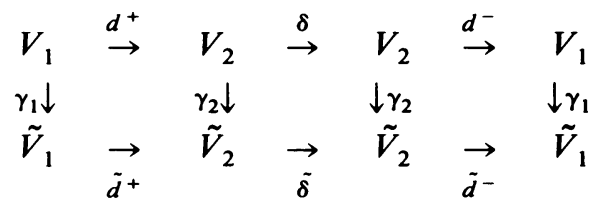

If for some infinitesimal character $\lambda$ to $\mathrm{Sl}(2, \mathrm{C}), H(\lambda)$ contains a finite-dimensional representation it contains exactly 2 irreducible representations. In this case $H(\lambda)$ is equivalent to $\mathfrak{X}$. For all other infinitesimal characters of $\mathrm{Sl}(2, \mathrm{C}), H(\lambda)$ is equivalent to a genuine subcategory of $\mathfrak{X}$.

In this article we prove that part of this result is true in more generality.

Received by the editors August 3, 1978 and, in revised form, February 21, 1980.

1980 Mathematics Subject Classification. Primary 22E46; Secondary 20G05.

${ }^{1}$ Research partially supported by National Science Foundation Grant NSF MCS 77-02053. 
Let $G$ be a semisimple connected linear Lie group with Lie algebra $g, U(g)$ the enveloping algebra of $g$ and $\pi_{1}, \pi_{2}$ irreducible Harish-Chandra modules of $U(\mathrm{~g})$. The category of all indecomposable Harish-Chandra modules, whose irreducible composition factors are equivalent to $\pi_{1}, \pi_{2}$ is denoted by $H\left(\pi_{1}, \pi_{2}\right)$.

THEOREM 1. Assume $\pi_{1}$ is an irreducible finite-dimensional representation of $G$ and $\pi_{2}$ is an irreducible representation so that

$$
\operatorname{Ext}_{U(\mathrm{~g})}^{1}\left(\pi_{1}, \pi_{2}\right) \neq 0 .
$$

Then $H\left(\pi_{1}, \pi_{2}\right)$ is isomorphic to a subcategory of $\mathfrak{X}$.

To prove this result we consider in the first part of the article a more general situation. We consider a category $H\left(\pi_{1}, \pi_{2}\right)$ where $\pi_{1}, \pi_{2}$ are irreducible representations with regular infinitesimal character such that

$$
\begin{gathered}
\operatorname{dim} \operatorname{Ext}_{U(\mathrm{~g})}^{1}\left(\pi_{1}, \pi_{2}\right)=1, \quad \operatorname{dim} \operatorname{Ext}_{U(\mathrm{~g})}^{1}\left(\pi_{2}, \pi_{2}\right)<1, \\
\operatorname{dim} \operatorname{Ext}_{U(\mathrm{~g})}^{1}\left(\pi_{1}, \pi_{1}\right)=0
\end{gathered}
$$

and such that there are representations $\delta_{i}, i=1,2$, of the maximal compact subgroup $K$ of $G$ such that

$$
\operatorname{dim} \operatorname{Hom}_{K}\left(\delta_{i}, \pi_{j}\right)=\delta_{i j} .
$$

We construct a functor from the category $H\left(\pi_{1}, \pi_{2}\right)$ into the category $\mathfrak{X}$ as follows. Let $H\left(\delta_{1}\right), H\left(\delta_{2}\right)$ be the subspaces of the isotypic components of $(\pi, H) \in$ $H\left(\pi_{1}, \pi_{2}\right)$ which transform according to the highest weights of $\delta_{1}, \delta_{2}$. We construct $X_{21}, X_{21}, X_{2} \in U(\mathfrak{g})$ with

$$
\begin{gathered}
\pi\left(X_{21}\right): H\left(\delta_{1}\right) \rightarrow H\left(\delta_{2}\right), \quad \pi\left(X_{12}\right): H\left(\delta_{2}\right) \rightarrow H\left(\delta_{1}\right), \\
\pi\left(X_{2}\right): H\left(\delta_{2}\right) \rightarrow H\left(\delta_{2}\right)
\end{gathered}
$$

so that

$$
\left[H\left(\delta_{1}\right), H\left(\delta_{2}\right), \pi\left(X_{21}\right)_{\mid H\left(\delta_{1}\right)}, \pi\left(X_{12}\right)_{\mid H\left(\delta_{2}\right)}, \pi\left(X_{2}\right)_{\mid H\left(\delta_{2}\right)}\right]
$$

is an object of $\mathfrak{X}$. Then we show that under these assumptions $H\left(\pi_{1}, \pi_{2}\right)$ is equivalent to a subcategory of $\mathfrak{X}$.

In the second part of the article we study extensions of finite-dimensional representations by irreducible infinite-dimensional ones and prove

TheOREM 2. Assume $\pi_{1}$ is a finite-dimensional irreducible representation of $G, \pi_{2}$ is an irreducible infinite-dimensional one.

(a) $\operatorname{dim} \operatorname{Ext}_{U(\mathrm{~g})}^{1}\left(\pi_{1}, \pi_{2}\right)=\operatorname{dim} \operatorname{Ext}_{U(\mathrm{~g})}^{1}\left(\pi_{2}, \pi_{1}\right)=1$.

(b) If $\operatorname{dim} \operatorname{Ext}_{U(g)}^{1}\left(\pi_{1}, \pi_{2}\right)=1$, every nontrivial extension of $\pi_{1}$ by $\pi_{2}$ is equivalent to a subrepresentation of a principal series representation.

(c) Under the assumptions of (b) $\operatorname{dim} \operatorname{Ext}_{U(\mathfrak{g})}^{1}\left(\pi_{2}, \pi_{1}\right) \leqslant 1$.

So the assumptions of the first part are satisfied if we choose for $\pi_{1}$ the one-dimensional representation of $G$ and for $\pi_{2}$ an irreducible representation with $\operatorname{Ext}_{U(g)}^{1}\left(\pi_{1}, \pi_{2}\right) \neq 0$. So we conclude that $H\left(\pi_{1}, \pi_{2}\right)$ is equivalent to a subcategory of $\mathfrak{X}$. Now Theorem 1 follows using tensoring functors. 
The author would like to thank D. Vogan for many helpful suggestions as well as I. E. Segal for his interest in an early stage of this work.

1. Notations and definitions. Let $G$ be a connected semisimple Lie group with finite center, $K$ its maximal compact subgroup, $P=$ MAN a minimal parabolic subgroup. We denote the Lie algebra of a subgroup of $G$ by the corresponding small German letter. The complex dual of a subalgebra $g_{1}$ is denoted by $g_{1}^{\prime}$.

A representation $\pi: G \rightarrow$ Aut $H, H$ a Banach space, is said to have length $n$ if there is a chain of closed $\pi$-invariant subspaces $0=H_{0} \subset H_{1} \subset \cdots \subset H_{n}=H$ such that the representation on $H_{i} / H_{i-1} \neq 0$ is irreducible. For each representation $\pi: G \rightarrow$ Aut $H$ we call the representation of the enveloping algebra $U(\mathrm{~g})$ of $\mathrm{g}$ on the space of $K$-finite vectors the associated Harish-Chandra module, which will be denoted also by $(\pi, H)$ or sometimes simply by $\pi$.

The set of equivalence classes of irreducible representations of $K$ is denoted by $\hat{K}$. We call $\delta \in \hat{K}$ a $K$-type of the Harish-Chandra module $\pi$ if for $\tau_{\delta} \in \delta$, $\operatorname{Hom}_{K}\left(\tau_{\delta}, \pi\right) \neq 0$.

A compatible $(\mathrm{g}, K)$ module is called admissible if

(a) it is finitely generated as a $U(\mathrm{~g})$ module,

(b) $\operatorname{dim} \operatorname{Hom}_{K}\left(\tau_{\delta}, \pi\right)<\infty$ for all $\tau_{\delta} \in \delta \in \hat{K}$.

By a theorem of W. Casselman [2] each admissible ( $g, K)$ module is equivalent to a Harish-Chandra module associated to a representation of $G$ of finite length. So we will not distinguish between representations of finite length and admissible $(\mathfrak{g}, K)$ modules.

Let $\pi: G \rightarrow$ Aut $H$ be a representation of finite length. It is said to be indecomposable if it is not equivalent to a direct sum of representations. The maximal subrepresentation, which decomposes into a direct sum of irreducible representations, is called the socle of $\pi_{1}$. Let $0=H_{0} \subset H_{1} \subset \cdots \subset H_{m}=H$ be a chain of closed $\pi$-invariant subspaces such that $H_{i} / H_{i-1}$ is the socle of the representation on $H / H_{i-1}$. We call $H_{i} / H_{i-1}$ the ith floor of $\pi$ and $m$ the height of $\pi$. It is an invariant of the representation.

Let $\pi_{1}, \pi_{2}$ be irreducible representations of $G$. A representation $\pi$ of length 2 is called an extension of $\pi_{1}$ by $\pi_{2}$ if $\pi_{1}$ and $\pi_{2}$ are equivalent to composition factors of $\pi$ and if $\pi_{1}$ is a subrepresentation of $\pi$. The extension $\pi$ is nontrivial if $\pi$ is indecomposable. We call $\pi$ an $m$-fold self-extension of an irreducible representation $\pi_{0}$ if $\pi$ is indecomposable, has length $m$ and all its composition factors are equivalent to $\pi_{0}$.

For any two irreducible Harish-Chandra modules $\pi_{1}, \pi_{2}$ we define $\operatorname{Ext}_{U_{(\mathrm{g})}}^{1}\left(\pi_{1}, \pi_{2}\right)$ as the group of equivalence classes of short exact sequences $0 \rightarrow \pi_{1} \rightarrow \pi \rightarrow \pi_{2} \rightarrow 0$ as in [1]. Denote by $e\left(\pi_{1}, \pi_{2}\right)$ the number of equivalence classes of representations which are extensions of $\pi_{1}$ by $\pi_{2}$.

1.1. LeMma. If $\operatorname{dim} \operatorname{Ext}_{U(\mathfrak{g})}^{1}\left(\pi_{1}, \pi_{2}\right)=1$, then

$$
e\left(\pi_{1}, \pi_{2}\right)=\operatorname{dim} \operatorname{Ext}_{U(g)}^{1}\left(\pi_{1}, \pi_{2}\right)=1 .
$$


Proof. Let $\pi: G \rightarrow$ Aut $H$ be a nontrivial extension of $\left(\pi_{1}, H_{1}\right)$ by $\left(\pi_{2}, H_{2}\right)$. We may assume $H_{1} \subset H$. Let $V$ be a subspace of $H$ so that

(a) $U(\mathrm{~g}) V$ is dense in $H$,

(b) $V \cap H_{1} \neq 0$,

(c) $\operatorname{dim} V=2$,

(d) $V$ is $K$-finite.

Let $U(\mathrm{~g})^{V}$ be the stabilizer of $V$ in $U(\mathrm{~g})$. We can choose a basis $v_{1}, v_{2}$ of $V$ so that $U(\mathrm{~g})^{V}$ acts as upper triangular matrices. For $\lambda \in \mathbf{C} \backslash 0$ put

$$
T_{\lambda}=\left(\begin{array}{ll}
1 & 0 \\
0 & \lambda
\end{array}\right) \text { and }\left.\pi_{\lambda}\right|_{V}=\left.T_{\lambda} \pi\right|_{V} T_{\lambda}^{-1}
$$

$\pi_{\lambda \mid V}$ is a representation of $U(\mathfrak{g})^{V}$ and extends to an admissible representation $\pi_{\lambda}$ of $U(\mathrm{~g})$, which is equivalent to $\pi$. On the other hand, the exact sequences

$$
0 \rightarrow \pi_{1} \rightarrow \pi \rightarrow \pi_{2} \rightarrow 0, \quad 0 \rightarrow \pi_{1} \rightarrow \pi_{\lambda} \rightarrow \pi_{2} \rightarrow 0
$$

are not equivalent. Q.E.D.

Let $\mathfrak{h}_{\mathbf{C}} \subset \mathfrak{g} \otimes \mathbf{C}$ be a Cartan subalgebra, $\Sigma$ the roots of $\left(\mathfrak{h}_{\mathbf{C}}, \mathfrak{g} \otimes \mathbf{C}\right), \Sigma^{+}$the positive roots and $\mathrm{C}^{+}$the corresponding dominant Weyl chamber. Write $W_{\mathbf{C}}=$ $W\left(\mathfrak{g} \otimes \mathbf{C}, \mathfrak{h}_{\mathbf{C}}\right)$ for the Weyl group of $\Sigma$.

Let $Z(\mathfrak{g})$ denote the center of $U(\mathfrak{g})$. If $S\left(\mathfrak{h}_{\mathbf{C}}\right)$ is the symmetric algebra of $\mathfrak{h}_{\mathbf{C}}$, then Harish-Chandra has defined an algebra isomorphism $\xi: Z(\mathfrak{g}) \rightarrow S\left(\mathfrak{h}_{\mathbf{C}}\right)^{W_{\mathbf{c}}}$. We say an admissible module $\pi$ has infinitesimal character $\gamma$, if $z \in Z(g)$ acts by the scalar $\xi(z)(\gamma)$ and $\gamma$ is contained in the closed Weyl chamber $\overline{\mathcal{C}}^{+}$. The representation is said to have singular infinitesimal character if $\gamma \in \bar{\complement}^{+} \backslash \mathrm{e}^{+}$. Otherwise the infinitesimal character is called regular.

We recall from [1] the

1.2. Lemma. Assume $\pi_{1}, \pi_{2}$ are irreducible representations with different infinitesimal characters. Then

$$
\operatorname{Ext}_{U(g)}^{1}\left(\pi_{1}, \pi_{2}\right)=0
$$

2. The category $H\left(\pi_{1}, \pi_{2}\right)$. Let $\pi_{1}, \pi_{2}$ be irreducible inequivalent representations of $G$. We write $H\left(\pi_{1}, \pi_{2}\right)$ for the abelian category which has as objects HarishChandra modules, whose composition factors are either isomorphic to $\pi_{1}$ or to $\pi_{2}$. The morphisms are homomorphisms between Harish-Chandra modules.

We associate to $\pi_{1}, \pi_{2}$ a graph as follows. We represent $\pi_{1}$ and $\pi_{2}$ by dots and join $\pi_{i}$ with $\pi_{j}$ by $\operatorname{dim} \operatorname{Ext}_{U(g)}^{1}\left(\pi_{i}, \pi_{j}\right)$ arrows. Below each dot we write the number of the representation it represents and write $T(i, j)$ for the arrows from $\operatorname{dot}(i)$ to $\operatorname{dot}(j)$.

Define a representation of a graph to be a map which maps each dot into a finite-dimensional complex vector space and each arrow into a linear map between the corresponding vector spaces.

If $V=\left\{V_{1}, V_{2}, A(t)\right\}$ and $W=\left\{W_{1}, W_{2}, B(t)\right\}$ are two representations we define a morphism from $V$ to $W$ to be a pair $\left(C_{1}, C_{2}\right)$ with $C_{i}: V_{i} \rightarrow W_{i}$ so that for 
$t \in T(i, j)$ the diagram

commutes.

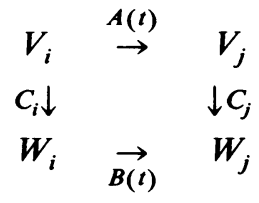

The category of representations of a graph is an abelian category [3], [13]. Hence direct summands, subrepresentations, Jordan-Hölder series, length, height and irreducibility are defined.

We call the sum of the dimensions of the two vectorspaces the dimension of the representation.

Now assume for this section that

(A) the infinitesimal character of $\pi_{2}$ is regular,

(B) $\operatorname{dim} \operatorname{Ext}_{U(\mathrm{~g})}^{1}\left(\pi_{1}, \pi_{2}\right)=\operatorname{dim} \operatorname{Ext}_{U(\mathrm{~g})}^{1}\left(\pi_{2}, \pi_{1}\right)=1$,

(C) $\operatorname{dim} \operatorname{Ext}_{U(\mathrm{~g})}^{1}\left(\pi_{2}, \pi_{2}\right) \leqslant 1$,

(D) $\operatorname{dim} \operatorname{Ext}_{U(g)}^{1}\left(\pi_{1}, \pi_{1}\right)=0$,

(E) there exist $\delta_{i} \in \hat{K}, i=1,2$, so that for $\tau_{\delta_{i}} \in \delta_{i}$

$$
\operatorname{dim} \operatorname{Hom}_{K}\left(\tau_{\delta_{i}}, \pi_{j}\right)=\delta_{i j} \text {. }
$$

Our first main result will be that under these assumptions $H\left(\pi_{1}, \pi_{2}\right)$ is isomorphic to a subcategory of the representations of the graph $D$

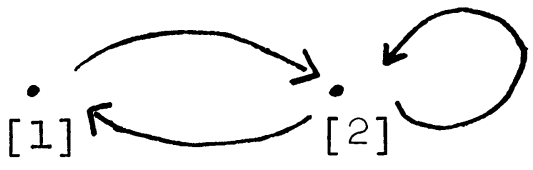

associated to $\left(\pi_{1}, \pi_{2}\right)$.

Let $\delta_{1}, \delta_{2} \in \hat{K}$ satisfying $E$. For $(\pi, H) \in H\left(\pi_{1}, \pi_{2}\right)$ denote by $H_{\delta_{1}}, H_{\delta_{2}}$ the corresponding isotypic components of $\pi$. Let $n_{k}$ be the nilradical of a Borel subalgebra $\mathfrak{b}_{k}=\mathfrak{h}_{k, c} \oplus \mathfrak{e}_{k}$ of $\mathfrak{t} \oplus \mathbf{C}, \lambda\left(\delta_{1}\right), \lambda\left(\delta_{2}\right) \in \mathfrak{h}_{k_{\mathbf{c}}}$ the highest weights of $\delta_{1}, \delta_{2}$ and $H\left(\delta_{1}\right), H\left(\delta_{2}\right)$ the corresponding highest weight spaces of $H_{\delta_{1}}, H_{\delta_{2}}$. Obviously the multiplicity of $\pi_{i}$ in the Jordan-Hölder series of $\pi$ is equal to the dimension of $H\left(\delta_{i}\right)$.

For $\delta \in K$ write $I^{\delta}$ for the kernel of $\delta$ in $U(\mathfrak{f})$ and for $\delta, \sigma \in \hat{K}$ put

$$
U(\mathfrak{g})^{\delta \sigma}=\left(u \in U(\mathfrak{g}) \mid I^{\delta} u \in U(\mathfrak{g}) I^{\sigma}\right) .
$$

2.1. Lemma. Let $\pi$ be a nontrivial extension of $\pi_{i}$ by $\pi_{j}, i, j=1,2, i \neq j$. There exists a (nonunique) $X^{i j} \in U(\mathfrak{g})^{\delta_{i} \delta_{j}}$ with

$$
\pi\left(X^{i j}\right): H\left(\delta_{j}\right) \rightarrow H\left(\delta_{i}\right) .
$$

Proof. Since $\pi$ is generated by $H\left(\delta_{j}\right)$ there exists at least one $X^{i j} \in U(\mathfrak{g})^{\delta_{i} \delta_{j}}$ with this property. Q.E.D.

2.2. Proposition. There exists at least one $X^{i j} \in U(\mathrm{~g})^{\delta_{i} \delta_{j}}$ so that for all $(\pi, H) \in$ $H\left(\pi_{1}, \pi_{2}\right), \pi\left(X^{i j}\right): H\left(\delta_{j}\right) \rightarrow H\left(\delta_{i}\right)$. 
Proof. The Cartan subalgebra $\mathfrak{h}_{\mathbf{c}}$ acts semisimply on $\mathfrak{g}_{\mathbf{c}}$ and $U(\mathfrak{g})$. We may choose an $X \in U(\mathrm{~g})^{\delta_{i}, \delta_{j}}$ satisfying Lemma 2.1 which is contained in the eigenspace for the eigenvalue $\lambda=\lambda\left(\delta_{j}\right)-\lambda\left(\delta_{i}\right)$. Q.E.D.

We fix this $X^{i j} \in U(\mathrm{~g})$ once and for all and call it $X_{i j}$.

2.3. Lemma [16, Proposition 6.1]. Let $(\pi, H)$ be an irreducible representation of $G$ with regular infinitesimal character. Suppose $\operatorname{dim} \operatorname{Ext}_{U(\mathrm{~g})}^{1}(\pi, \pi)=1$. Let $(\tilde{\pi}, H)$ be a nontrivial extension of $\pi$ by $\pi$. There exists an $X \in Z(g)$ which does not act semisimply on $\tilde{\pi}$.

If $\operatorname{Ext}_{U(\mathfrak{g})}^{1}\left(\pi_{2}, \pi_{2}\right) \neq 0$, we choose such an $X \in Z(\mathrm{~g})$ once and for all and call it $X_{2}$. If $\tilde{\pi}_{2}$ is any nontrivial extension of $\pi_{2}$ by $\pi_{2}$, then $X_{2}$ does not act semisimply on $H\left(\delta_{2}\right)$. If $\operatorname{Ext}_{U(\mathrm{~g})}^{1}\left(\pi_{2}, \pi_{2}\right)=0$ put $X_{2}=I \in U(\mathfrak{g})$.

As in Proposition 2.2 we show

2.4. Lemma. Let $\pi \in H\left(\pi_{1}, \pi_{2}\right)$. Then $\pi\left(X_{2}\right): H\left(\delta_{2}\right) \rightarrow H\left(\delta_{2}\right)$.

We define a functor $\psi$ from $H\left(\pi_{1}, \pi_{2}\right)$ into the category of representations of $D$ by

$$
\pi \rightarrow\left(H\left(\delta_{1}\right), H\left(\delta_{2}\right), \pi\left(X_{21}\right)_{\mid H\left(\delta_{1}\right)}, \pi\left(X_{12}\right)_{\mid H\left(\delta_{2}\right)}, \pi\left(X_{2}\right)_{\mid H\left(\delta_{2}\right)}\right) .
$$

The functor $\psi$ is a functor between abelian categories and we will show next that it is a monomorphism.

If $\pi \in H\left(\pi_{1}, \pi_{2}\right)$ we write $\pi_{s}$ for the socle of $\pi$ and $\psi(\pi)_{s}$ for the socle of $\psi(\pi)$. Obviously $\psi\left(\pi_{s}\right) \subset \psi(\pi)_{s}$.

\subsection{LEMMA. $\psi\left(\pi_{s}\right)=\psi(\pi)_{s}$.}

Proof. We prove this by inductions on the height of $\pi$. Let $\pi$ be a representation of height $n, \pi=\bigoplus_{j=1}^{l} \pi^{j}$, where $\pi^{j}$ is indecomposable of height $\leqslant n$. Then $\psi(\pi)=\bigoplus_{j=1}^{l} \psi\left(\pi_{s}^{j}\right)$. Since $\pi_{s}=\bigoplus_{j=1}^{l} \pi_{s}^{j}$ and $\psi(\pi)_{s}=\bigoplus_{j=1}^{l} \psi\left(\pi_{s}^{j}\right)$ we may assume that $\pi$ is indecomposable.

Assume now $\pi$ is a representation of height 2 and let $v \in H\left(\delta_{1}\right) \oplus H\left(\delta_{2}\right), v \notin \pi_{s}$. We may assume that $v$ is contained in a subrepresentation $\pi^{1}$ of $\pi$ and that $\pi^{1} / \pi_{s}^{1}$ is irreducible. But $\pi^{1}$ is a subrepresentation of a direct sum of representations of length 2 . So by Lemmas 2.1 and $2.3 v \notin \psi(\pi)_{s}$.

Let $\pi$ be an indecomposable representation of height $n$. Assume that there exists a subrepresentation $V \neq 0$ of $\psi(\pi)$, so that $\psi\left(\pi_{s}\right) \oplus V \subset \psi(\pi)_{s}$. But then

$$
V \subset\left(\psi(\pi) / \psi\left(\pi_{s}\right)\right)_{s}=\left(\psi\left(\pi / \pi_{s}\right)\right)_{s}
$$

and by the induction hypothesis $V \subset \psi\left(\left(\pi / \pi_{s}\right)_{s}\right)$. Let $\pi^{1}$ be a subrepresentation of $\pi$ such that $\pi_{s} \hookrightarrow \pi^{1}$ and

$$
\pi^{1} / \pi_{s}=\left(\pi / \pi_{s}\right)_{s}
$$

Hence $V \subset \psi\left(\pi^{1} / \pi_{s}\right)=\psi\left(\pi^{1}\right) / \psi\left(\pi_{s}\right)$ and $V \subset \psi\left(\pi^{1}\right)$. Since $\pi^{1}$ has height 2 and $\pi_{s}$ is the socle of $\pi^{1}$ we have a contradiction to the first inductive step. Hence $V=0$. Q.E.D.

2.6. Lemma. Let $V$ be a subrepresentation of $\psi(\pi), \pi \in H\left(\pi_{1}, \pi_{2}\right)$. There exists a subrepresentation $\tilde{\pi}$ of $\pi$ such that $\psi(\tilde{\pi})=V$. 
Proof. Here we use induction on the length of $V$. If $V$ has length 1 , the lemma follows from the definitions. Assume that $V$ has length $n$. Let $V^{\prime}$ be an irreducible subrepresentation of $V_{s}$ and $\pi^{\prime}$ a subrepresentation of $\pi$ such that $\psi\left(\pi^{\prime}\right)=V^{\prime}$. Then

$$
0 \rightarrow \pi^{\prime} \rightarrow \pi \rightarrow \pi / \pi \rightarrow 0
$$

and

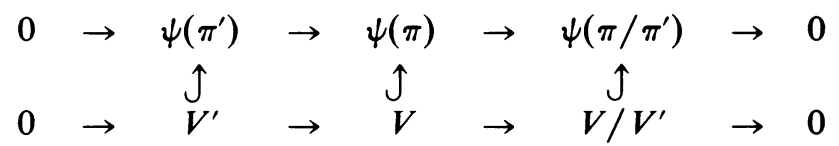

Let $\pi^{\prime \prime}$ be the subrepresentation of $\pi / \pi^{\prime}$ so that $\psi\left(\pi^{\prime \prime}\right)=V / V^{\prime}$ and let $\pi_{V}$ be the inverse image of $\pi^{\prime}$ in $\pi$. Then $\psi\left(\pi_{V}\right) \supset V^{\prime}$ and $\psi\left(\pi_{V}\right) / V^{\prime}=V / V^{\prime}$. Thus $\psi\left(\pi_{V}\right)=$ V. Q.E.D.

2.7. THEOREM. The functor $\psi$ maps indecomposable representations in $H\left(\pi_{1}, \pi_{2}\right)$ to indecomposable representations in $D$.

Proof. Let $\pi \in H\left(\pi_{1}, \pi_{2}\right)$. Assume $\psi(\pi)=V_{1} \oplus V_{2}$. There exist subrepresentations $\pi^{1}$ and $\pi^{2}$ of $\pi$ so that $\psi\left(\pi^{1}\right)=V_{1}, \psi\left(\pi^{2}\right)=V_{2}$. Then $\pi^{1} \cup \pi^{2}=\pi$ since the length of the subrepresentation $\pi^{1} \cup \pi^{2}$ is equal to the length of $\pi$.

On the other hand, $\pi^{1} \cap \pi^{2}=\{0\}$ since

$$
\psi\left(\pi^{1} \cap \pi^{2}\right)=\psi\left(\pi^{1}\right) \cap \psi\left(\pi^{2}\right)=V_{1} \cap V_{2}=0 .
$$

So $\pi=\pi_{1} \oplus \pi_{2}$. Q.E.D.

2.8. THEOREM. The functor $\psi$ is a monomorphism.

Proof. We first make the following observation. Suppose there are short exact sequences

$$
\begin{aligned}
& 0 \rightarrow \pi^{1} \rightarrow \pi \rightarrow \pi^{2} \rightarrow 0, \\
& 0 \rightarrow \pi^{1} \rightarrow \bar{\pi} \rightarrow \pi^{2} \rightarrow 0
\end{aligned}
$$

with $\pi^{1}, \pi^{2} \in H\left(\pi_{1}, \pi_{2}\right)$. If the sequences

$$
\begin{aligned}
& 0 \rightarrow \psi\left(\pi^{1}\right) \rightarrow \psi(\pi) \rightarrow \psi\left(\pi^{2}\right) \rightarrow 0, \\
& 0 \rightarrow \psi\left(\pi^{1}\right) \rightarrow \psi(\bar{\pi}) \rightarrow \psi\left(\pi^{2}\right) \rightarrow 0
\end{aligned}
$$

are equivalent, then Theorem 2.7 and the fact that $\operatorname{Ext}_{U(\mathrm{~g})}^{1}\left(\pi^{1}, \pi^{2}\right)$ is a group imply that $S_{1}$ and $S_{2}$ are equivalent.

In particular, if $\pi$ and $\bar{\pi}$ have length 2 and $\psi(\pi) \cong \psi(\bar{\pi})$, then $\pi \cong \bar{\pi}$.

We now proceed by induction on the length of $\pi$. Let $\pi^{1}, \pi^{2} \in H\left(\pi_{1}, \pi_{2}\right)$ so that $\psi\left(\pi^{1}\right)=\psi\left(\pi^{2}\right)$. Then $\psi\left(\pi_{S}^{1}\right)=\psi\left(\pi_{S}^{2}\right)=\psi\left(\pi^{1}\right)_{S}=\psi\left(\pi^{2}\right)_{S}$. Since the length of $\pi^{1} / \pi_{S}^{1}$ and $\pi^{2} / \pi_{S}^{2}$ is smaller than the length of $\pi^{1}$ and $\pi^{2}$, and since

$$
\psi^{1}\left(\pi^{1}\right) / \psi\left(\pi_{S}^{1}\right) \cong \psi\left(\pi^{1} / \pi_{S}^{1}\right) \cong \psi\left(\pi^{2} / \pi_{S}^{2}\right)=\psi\left(\pi^{2}\right) / \psi\left(\pi_{S}^{2}\right)
$$

we deduce $\pi^{1} / \pi_{S}^{1} \cong \pi^{2} / \pi_{S}^{2}$. Consider now the sequences

$$
\begin{aligned}
& 0 \rightarrow \pi_{S}^{1} \rightarrow \pi^{1} \rightarrow \pi^{1} / \pi_{S}^{1} \rightarrow 0, \\
& 0 \rightarrow \pi_{S}^{2} \rightarrow \pi^{2} \rightarrow \pi^{2} / \pi_{S}^{2} \rightarrow 0 .
\end{aligned}
$$


Since by construction the sequences

$$
\begin{aligned}
& 0 \rightarrow \psi\left(\pi_{S}^{1}\right) \rightarrow \psi\left(\pi^{1}\right) \rightarrow \psi\left(\pi^{1} / \pi_{S}^{1}\right) \rightarrow 0, \\
& 0 \rightarrow \psi\left(\pi_{S}^{1}\right) \rightarrow \psi\left(\pi^{2}\right) \rightarrow \psi\left(\pi^{1} / \pi_{S}^{1}\right) \rightarrow 0
\end{aligned}
$$

are equivalent; the theorem follows. Q.E.D.

2.9. Corollary. Let $\pi^{1}, \pi^{2} \in H\left(\pi_{1}, \pi_{2}\right)$. Assume $\psi\left(\pi^{1}\right)$ and $\psi\left(\pi^{2}\right)$ are equivalent. Then $\pi^{1}$ and $\pi^{2}$ are equivalent as well.

Proof. We make use of the

2.10. Lemma. Let $\pi: U(\mathrm{~g}) \rightarrow$ End $H$ be a Harish-Chandra module and $W \subset H$ be a subspace generating $H$. Denote by $U(\mathrm{~g})^{W}$ the stabilizer of $W$ in $U(\mathrm{~g})$. Let $W^{\prime}$ be another $U(\mathrm{~g})^{W}$-module and $A_{W}$ a surjective intertwining operator from $W$ onto $W^{\prime}$. Then $A_{W}$ extends to an intertwining operator

$$
A: \pi \rightarrow U(\mathfrak{g}) \underset{U(\mathfrak{g})}{\otimes} W^{\prime} .
$$

The proof of the lemma is straightforward and left to the reader.

We now return to the proof of the corollary. Let $\pi^{1}: U(\mathrm{~g}) \rightarrow$ End $H^{1}$ and $\pi^{2}: U(\mathrm{~g}) \rightarrow$ End $H^{2}$ be admissible representations of $U(\mathrm{~g})$. Put $W^{1}=H^{1}\left(\delta_{1}\right) \oplus$ $H^{1}\left(\delta_{2}\right)$ and $W^{2}=H^{2}\left(\delta_{1}\right) \oplus H^{2}\left(\delta_{2}\right)$. The intertwining operator $A: \psi\left(\pi_{1}\right) \rightarrow \psi\left(\pi_{2}\right)$ induces a linear operator $A_{W}: W^{1} \rightarrow W^{2}$. The algebra $U(\mathrm{~g})^{W^{1}}$ acts on $W^{1}$ by $\pi^{1}$ and we define an action $\pi_{A}$ on $W^{2}$ by $\pi_{A}=A_{W} \pi^{1} A_{W}^{-1}$. Since $A_{W}$ intertwines $\pi$ and $\pi_{A}$, by the lemma there is an intertwining operator

$$
A: \pi^{1} \rightarrow U(\mathrm{~g}) \underset{U(\mathrm{~g})}{\bigotimes_{W^{1}}} W^{2} .
$$

Put $\pi(A)=U(\mathfrak{g}) \otimes_{U(\mathfrak{g})^{w_{1}}} W^{2}$. By Theorem 2.8 it suffices to show $\psi\left(\pi_{2}\right)=\psi(\pi(A))$. But by construction

$$
\psi(\pi(A))=A\left(\psi\left(\pi^{1}\right)\right)=A\left(\psi\left(\pi^{1}\right)\right)=\psi\left(\pi_{2}\right) \text {. Q.E.D. }
$$

2.11. Corollary. There is a one-to-one correspondence of equivalence classes of Harish-Chandra modules in $H\left(\pi_{1}, \pi_{2}\right)$ and equivalence classes of representations in $\psi\left(H\left(\pi_{1}, \pi_{2}\right)\right)$.

Proof. This follows from the remarks after Lemmas 2.4 and 2.10. Q.E.D.

REMARK. In the proof of Corollary 2.11 we used only that $X_{2}$ satisfies the following conditions:

(a) if $\pi \in H\left(\pi_{1}, \pi_{2}\right)$, then $\pi\left(X_{2}\right): H\left(\delta_{2}\right) \rightarrow H\left(\delta_{2}\right)$,

(b) if $\pi$ is a nontrivial self-extension of $\pi_{2}$, then $\pi\left(X_{2}\right)_{\mid H\left(\delta_{2}\right)}$ is not semisimple.

3. A classification result. Let $\pi_{1}, \pi_{2}$ be irreducible representations of $G$ satisfying the conditions (A)-(E). Using properties of $X_{12}, X_{21}$ and $X_{2}$ we describe the image of the functor $\psi$ more closely.

3.1. LemMa. Let $(\pi, H) \in H\left(\pi_{1}, \pi_{2}\right)$. The operators

$$
\pi\left(X_{12} X_{21}\right)_{\mid H\left(\delta_{1}\right)} \text { and } \pi\left(X_{21} X_{12}\right)_{\mid H\left(\delta_{2}\right)}
$$

are nilpotent. 
Proof. Assume $X_{12} X_{21}$ does not act nilpotently on $H\left(\delta_{1}\right)$. Then it has at least one eigenvector $v_{1}$ with eigenvalue $\lambda \neq 0$. Let $W_{1}$ be the submodule generated by $v_{1}$ and let $W_{2}$ be the submodule of $W_{1}$ generated by $v_{2}=\pi\left(X_{21}\right) v_{1}$. Since

$$
\pi\left(X_{12}\right) \pi\left(X_{21}\right) v_{1}=\lambda v_{1} \in W_{1}
$$

we have $W_{1}=W_{2}$.

Let $W$ be a maximal invariant subspace of $W_{1}$ which does not contain $v_{1}$ and hence not $v_{2}$. So $W_{1} / W$ has composition factors equivalent to $\pi_{1}$ and $\pi_{2}$.

Assume there exists an invariant subspace $W^{1}$ so that $W \subset W^{1} \subset W_{1}$ and $W^{1} / W$ is isomorphic to $\pi_{2}$. So $v_{1} \notin W^{1}$, and since $W^{1}$ is invariant $v_{2} \notin W^{1}$. But this contradicts the choice of $W_{1}$.

By the same argument we show that $W_{1} / W$ has no subrepresentation isomorphic to $\pi_{1}$.

Since $\pi$ and hence $W_{1} / W$ have finite length, we get a contradiction. Q.E.D.

Let $\lambda$ be the infinitesimal character of $\pi_{2}$. Put $\lambda\left(\pi_{2}\right)=\psi\left(\lambda_{2}\right)\left(X_{2}\right)$. Since $X_{2}$ $\lambda\left(\pi_{2}\right)$ acts nilpotently on $\left(\pi^{\prime}, H\right) \in H\left(\pi_{1}, \pi_{2}\right)$, its restriction to $H\left(\delta_{2}\right)$ is nilpotent.

Let $0=H_{0} \subset \cdots \subset H_{l}=H$ be a maximal proper chain of $U(\mathrm{~g})$-invariant subspaces. Its intersection with $H\left(\delta_{1}\right)$ and $H\left(\delta_{2}\right)$ defines chains

$$
\begin{aligned}
& 0=H\left(\delta_{1}\right)_{0} \subset \cdots \subset H\left(\delta_{1}\right)_{l}=H\left(\delta_{1}\right), \\
& 0=H\left(\delta_{2}\right)_{0} \subset \cdots \subset H\left(\delta_{2}\right)_{l}=H\left(\delta_{2}\right) .
\end{aligned}
$$

After changing the enumeration we may assume that $H\left(\delta_{i}\right)_{j}$ is a nontrivial subspace of $H\left(\delta_{i}\right)_{j+1}$. We may choose a basis $v_{1}, \ldots, v_{s}$ of $H\left(\delta_{1}\right)$ so that $\left\langle v_{1}, \ldots, v_{j}\right\rangle=$ $H\left(\delta_{1}\right)_{j}$ and a basis $w_{1}, \ldots, w_{r}$ of $H\left(\delta_{2}\right)$ so that $\left\langle w_{1}, \ldots, w_{i}\right\rangle=H\left(\delta_{2}\right)_{i}$. We call this a basis according to the invariant subspace structure. Write

$$
\begin{gathered}
\bar{\pi}\left(X_{12}\right)=\pi\left(X_{12}\right)_{\mid H\left(\delta_{1}\right)}, \quad \bar{\pi}\left(X_{2}\right)=\pi\left(X_{2}\right)_{\mid H\left(\delta_{2}\right)}, \\
\bar{\pi}\left(X_{21}\right)=\pi\left(X_{21}\right)_{\mid H\left(\delta_{2}\right)} .
\end{gathered}
$$

Next we list some properties of representations in $H\left(\pi_{1}, \pi_{2}\right)$ which will be used later.

3.2. Lemma. (a) All indecomposable representations $\pi \in H\left(\pi_{1}, \pi_{2}\right)$ with $\operatorname{dim} H\left(\delta_{1}\right)$ $=2, \operatorname{dim} H\left(\delta_{2}\right)=1$ are equivalent; in particular $\psi(\pi)$ is equivalent to

$$
\left\{H\left(\delta_{1}\right), H\left(\delta_{2}\right), \bar{\pi}\left(X_{21}\right)=(1,0), \bar{\pi}\left(X_{12}\right)=\left(\begin{array}{l}
0 \\
1
\end{array}\right), \bar{\pi}\left(X_{2}\right)=\lambda\left(\pi_{2}\right)\right\} \text {. }
$$

(b) Assume there exists $\pi \in H\left(\pi_{1}, \pi_{2}\right)$ indecomposable, with $\operatorname{dim} H\left(\delta_{1}\right)=1$, $\operatorname{dim} H\left(\delta_{2}\right)=2$. Then $\psi(\pi)$ is equivalent to

$$
\left\{H\left(\delta_{1}\right), H\left(\delta_{2}\right), \bar{\pi}\left(X_{21}\right)=\left(\begin{array}{l}
0 \\
1
\end{array}\right), \bar{\pi}\left(X_{12}\right)=(1,0), \bar{\pi}\left(X_{2}\right)=\left(\begin{array}{cc}
\lambda\left(\pi_{2}\right) & 0 \\
\mu & \lambda\left(\pi_{2}\right)
\end{array}\right), \mu \in \mathbf{C}^{+}\right\} .
$$

(c) Assume there exists $\pi \in H\left(\delta_{1}, \delta_{2}\right)$, with $\operatorname{dim} H\left(\delta_{1}\right)=3, \operatorname{dim} H\left(\delta_{2}\right)=1$. Then $\pi$ is not indecomposable.

Proof. (a) and (b) follow from matrix computations.

To prove (b), it suffices to show that $\pi$ has a subrepresentation isomorphic to $\pi_{2}$. Assume $\pi$ has only one subrepresentation $\pi^{1}$ and $\pi^{1}$ is isomorphic to $\pi_{1}$. Since $\pi\left(X_{2}-\lambda\left(\pi_{2}\right)\right)$ is a nontrivial intertwining operator, its image is a subrepresentation 
of $\pi$. But $\pi_{1}$ is contained in the kernel of $\pi\left(X_{2}-\lambda\left(\pi_{2}\right)\right)$. Hence its image contains only subrepresentations isomorphic to $\pi_{2}$. So we get a contradiction. Now (b) follows by matrix computations. Q.E.D.

3.3A. TheOREM. Let $n \in \mathbf{N}$. There exist constants $\gamma=\gamma\left(\pi_{1}, \pi_{2}, \delta_{1}, \delta_{2}\right)$, $\gamma_{2}, \ldots, \gamma_{n-1}$ so that, if $\pi \in H\left(\pi_{1}, \pi_{2}\right)$ and length of $\pi=n$, then

$$
\bar{\pi}\left(X_{12}\right)\left\{\bar{\pi}\left(X_{2}\right)-\lambda\left(\pi_{2}\right) \mathrm{Id}-\gamma \bar{\pi}\left(X_{21} X_{12}\right)-\sum_{i=2}^{n-1} \gamma_{i}\left(\bar{\pi}\left(X_{2}\right)-\gamma\left(\pi_{2}\right) \mathrm{Id}\right)^{i}\right\}=0 .
$$

3.3B. Theorem. Let $n \in \mathbf{N}$. There exist constants $\mu=\mu\left(\pi_{1}, \pi_{2}, \delta_{1}, \delta_{2}\right)$, $\mu_{2}, \ldots, \mu_{n-1}$ so that, if $\pi \in H\left(\pi_{1}, \pi_{2}\right)$ and length of $\pi=n$, then

$$
\left\{\bar{\pi}\left(X_{2}\right)-\gamma\left(\pi_{2}\right) \mathrm{Id}-\mu \bar{\pi}\left(X_{21} X_{12}\right)-\sum_{i=2}^{n-1} \mu_{i}\left(\bar{\pi}\left(X_{2}\right)-\lambda\left(\pi_{2}\right) \mathrm{Id}\right)^{i}\right\} \bar{\pi}\left(X_{21}\right)=0 .
$$

Since the proofs of Theorems 3.3A and 3.3B are quite similar, we will give only the proof of Theorem 3.3A in detail. The proof is divided into a series of lemmas.

Assume for the moment that Theorem 3.3A holds for all representations of length shorter than $n$. Let $\pi$ be a representation of length $n-1$ and $\gamma$, $\gamma_{2}, \ldots, \gamma_{n-2} \in \mathbf{C}$ as in Theorem 3.3A. Suppose $\pi^{\prime}$ is a subrepresentation of $\pi$ of length $m<n-1$. Then

$$
\bar{\pi}^{\prime}\left(X_{12}\right)\left\{\bar{\pi}^{\prime}\left(X_{2}\right)-\gamma\left(\pi_{2}\right) \mathrm{Id}-\gamma \bar{\pi}^{\prime}\left(X_{21} X_{12}\right)-\sum_{i=2}^{n-2} \gamma_{i}\left(\bar{\pi}^{\prime}\left(X_{2}\right)-\gamma\left(\pi_{2}\right) \mathrm{Id}\right)^{i}\right\}=0 .
$$

Since $\bar{\pi}^{\prime}\left(X_{2}-\gamma\left(\pi_{2}\right)\right)^{i}=0$ for $i \geqslant m$ and since each representation of length $m$ is a subrepresentation of a representation of length $n-1$, the constants $\gamma$, $\gamma_{2}, \ldots, \gamma_{m-1}$ satisfy the conditions of Theorem 3.3A for representations of length $m<n-1$.

Let $\pi^{i} \in H\left(\pi_{1}, \pi_{2}\right), i=1,2$ with length of $\pi^{i}$ equal to $n_{i}<n . \pi^{0}=\pi^{1} \oplus \pi^{2}$ and assume $n_{1}+n_{2} \geqslant n$. Then

$$
\begin{aligned}
\bar{\pi}^{0}\left(X_{12}\right)\left\{\bar{\pi}^{0}\left(X_{2}\right)-\gamma\left(\pi_{2}\right) \mathrm{Id}-\gamma \bar{\pi}^{0}\left(X_{21} X_{12}\right)\right. \\
\\
\left.-\sum_{i=2}^{i=n_{1}-n_{2}-1} \gamma_{i}\left(\bar{\pi}^{0}\left(X_{2}\right)-\gamma\left(\pi_{2}\right) \mathrm{Id}\right)^{i}\right\}=0
\end{aligned}
$$

with arbitrary $\gamma_{i}, n \leqslant i<n_{1}+n_{2}$. Hence it suffices to prove Theorem 3.3A for indecomposable representations of length $n$.

If the length of $\pi$ is smaller than 4, then by Lemma 3.2(a), (b) Theorem 3.3A holds for all $\gamma \in(\mathrm{C} \backslash 0)$. So the theorem holds if $H\left(\pi_{1}, \pi_{2}\right)$ contains only indecomposable representations of length smaller than 4 . We assume therefore from now on that $H\left(\pi_{1}, \pi_{2}\right)$ contains indecomposable representations of length larger than 3 .

We first state a number of reduction techniques. Assume Theorem 3.3A holds for $n-1$. 
3.4. LemmA. Assume there exists $\pi \in H\left(\pi_{1}, \pi_{2}\right)$ indecomposable and of length $n$. Assume furthermore, that there exist submodules $\pi^{\prime}$ and $\pi^{\prime \prime}$ of length shorter than $n$, so that $\pi=\pi^{\prime} \cup \pi^{\prime \prime}$. Let $\gamma, \gamma_{2}, \ldots, \gamma_{n-1}$ be as in Theorem 3.3A. Then for each $\gamma_{n-1} \in \mathbf{C} \backslash 0$

$$
\bar{\pi}\left(X_{12}\right)\left\{\bar{\pi}\left(X_{2}\right)-\lambda\left(\pi_{2}\right) \mathrm{Id}-\gamma \bar{\pi}\left(X_{21} X_{12}\right)-\sum_{i=2}^{n-1} \gamma_{i}\left(\bar{\pi}\left(X_{2}\right)-\lambda\left(\pi_{2}\right) \mathrm{Id}\right)^{i}\right\}=0 .
$$

Proof. Let $\pi_{0}^{\prime}, \pi_{0}^{\prime \prime}$ be the submodules isomorphic to $\pi^{\prime} \cap \pi^{\prime \prime}$ of $\pi^{\prime}$ and $\pi^{\prime \prime}$, respectively. There exists an isomorphism $\alpha: \pi_{0}^{\prime} \rightarrow \pi_{0}^{\prime \prime}$ such that $\pi$ is isomorphic to $\left(\pi^{\prime} \oplus \pi^{\prime \prime}\right) / \pi_{\alpha}$, where $\pi_{\alpha}=\left\{(x, \alpha x), x \in \pi_{0}^{\prime}\right\}$. Since the length of $\pi^{\prime}$ and $\pi^{\prime \prime}$ is smaller than $n$, for each $\gamma \in \mathbf{C}$

$$
\begin{aligned}
\overline{\pi^{\prime} \oplus \pi^{\prime \prime}}\left(X_{12}\right)\left\{\overline{\pi^{\prime} \oplus \pi^{\prime \prime}}\left(X_{2}\right)-\lambda\left(\pi_{2}\right) \mathrm{Id}-\gamma \overline{\pi^{\prime} \oplus \pi^{\prime \prime}}\left(X_{21} X_{12}\right)\right. \\
\left.-\sum_{i=2}^{n-1} \gamma_{i}\left(\overline{\pi^{\prime} \oplus \pi^{\prime \prime}}\left(X_{2}\right)-\lambda\left(\pi_{2}\right) \mathrm{Id}\right)^{i}\right\}=0
\end{aligned}
$$

and hence also for each quotient of $\pi^{\prime} \oplus \pi^{\prime \prime}$. Q.E.D.

3.5. Lemma. Assume there exists $\pi \in H\left(\pi_{1}, \pi_{2}\right)$ indecomposable and of length $n$. Assume furthermore that $\pi$ has not a unique subrepresentation. Let $\gamma, \gamma_{2}, \ldots, \gamma_{n-2}$ be as in Theorem 3.3A. Then for each $\gamma_{n-1} \in \mathbf{C}$

$$
\bar{\pi}\left(X_{12}\right)\left\{\bar{\pi}\left(X_{2}\right)-\lambda\left(\pi_{2}\right) \mathrm{Id}-\gamma \bar{\pi}\left(X_{21} X_{12}\right)-\sum_{i=2}^{n-1} \gamma_{i}\left(\bar{\pi}\left(X_{2}\right)-\lambda\left(\pi_{2}\right) \mathrm{Id}\right)^{i}\right\}=0 .
$$

Proof. There exist subrepresentations $\pi^{\prime}$ and $\pi^{\prime \prime}$ of $\pi, \pi_{s}^{\prime} \neq \pi_{s}^{\prime \prime}$, such that $\pi$ is isomorphic to a subrepresentation of $\pi^{\prime} \oplus \pi^{\prime \prime}$. Since the lemma holds for $\pi^{\prime} \oplus \pi^{\prime \prime}$, it holds also for each subrepresentation. Q.E.D.

3.6. Lemma. Assume there exists $\pi \in H\left(\pi_{1}, \pi_{2}\right)$ indecomposable and of length $n$. Assume furthermore, $\pi$ has a subrepresentation $\tilde{\pi}_{2}$ isomorphic to $\pi_{2}$. Let $\gamma$, $\gamma_{2}, \ldots, \gamma_{n-2}$ be as in Theorem 3.3A. Then for each $\gamma_{n-1} \in \mathbf{C}$

$$
\bar{\pi}\left(X_{12}\right)\left\{\bar{\pi}\left(X_{2}\right)-\lambda\left(\pi_{2}\right) \mathrm{Id}-\gamma \bar{\pi}\left(X_{21} X_{12}\right)-\sum_{i=2}^{n-1} \gamma_{i}\left(\bar{\pi}\left(X_{2}\right)-\lambda\left(\pi_{2}\right)\right)^{i}\right\}=0 .
$$

Proof. Choose a basis $v_{1}, \ldots, v_{l}$ of $H\left(\delta_{1}\right)$ and $w_{1}, \ldots, w_{r}$ of $H\left(\delta_{2}\right)$ according to the invariant subspace structure of $\pi$; we may assume $w_{r} \in \tilde{\pi}_{2}$. Put $\underline{\pi}=\pi / \tilde{\pi}_{2}$ and $\underline{w}_{i}, v_{j}$ for the corresponding projections of $w_{i}$ and $v_{i}$. Then by assumption

$$
\begin{gathered}
\underline{\pi}\left(X_{12}\right)\left\{\underline{\pi}\left(X_{2}\right)-\lambda\left(\pi_{2}\right) \mathrm{Id}-\sum_{i=2}^{n-2} \gamma_{i}\left(\underline{\pi}\left(X_{2}\right)-\gamma\left(\pi_{2}\right) \mathrm{Id}\right)^{i}\right\} \underline{w}_{i} \\
=\gamma \underline{\pi}\left(X_{12}\right) \underline{\pi}\left(X_{21}\right) \pi\left(X_{i 2}\right) \underline{w}_{i} \in H\left(\delta_{i}\right)
\end{gathered}
$$


and $\underline{\pi}\left(X_{2}-\lambda\left(\pi_{2}\right)\right)^{n-1} w_{i}=0, i=1, \ldots, r$. So for all $\gamma_{n-1} \in \mathbf{C}$

$$
\begin{gathered}
\underline{\pi}\left(X_{12}\right)\left\{\underline{\pi}\left(X_{2}\right)-\lambda\left(\pi_{2}\right) \mathrm{Id}-\sum_{i=2}^{n-1} \gamma_{i} \underline{\pi}\left(X_{2}-\lambda\left(\pi_{2}\right)\right)^{i}\right\} w_{i} \\
=\gamma \underline{\pi}\left(X_{12}\right) \underline{\pi}\left(X_{21}\right) \underline{\pi}\left(X_{12}\right) \underline{w}_{i} .
\end{gathered}
$$

On the other hand, since

$$
\pi\left(X_{2}-\lambda\left(\pi_{2}\right)\right)^{n-1} w_{i}=0 \text { for } i=2, \ldots, r .
$$

we have for all $\gamma_{n-1} \in \mathbf{C}$ and $i=2, \ldots, r$

$$
\begin{gathered}
\pi\left(X_{12}\right)\left\{\pi\left(X_{2}\right)-\lambda\left(\pi_{2}\right) \mathrm{Id}-\sum_{i=2}^{n-1} \gamma_{i}\left(\underline{\pi}\left(X_{2}\right)-\lambda\left(\pi_{2}\right) \mathrm{Id}\right)^{i}\right\} w_{i} \\
=\gamma \pi\left(X_{12}\right) \pi\left(X_{21}\right) \pi\left(X_{12}\right) w_{i},
\end{gathered}
$$

and for all $\gamma_{n-1} \in \mathbf{C}$

$$
\begin{aligned}
\pi\left(X_{12}\right)\left\{\pi\left(X_{2}\right)-\lambda\left(\pi_{2}\right) \mathrm{Id}-\right. & \left.\sum_{i=2}^{n-1} \gamma_{i}\left(\pi\left(X_{2}\right)-\lambda\left(\pi_{2}\right)\right)^{i}\right\} w_{1} \\
& =\gamma \pi\left(X_{12}\right) \pi\left(X_{21}\right) \pi\left(X_{12}\right) w_{1}+u
\end{aligned}
$$

where $u$ is contained in an invariant subspace in $\psi\left(\pi_{2}\right) \cap H\left(\delta_{2}\right)$. Since

$$
\pi\left(X_{12}\right)\left\{\pi\left(X_{2}\right)-\lambda\left(\pi_{2}\right) \mathrm{Id}-\sum_{i=2}^{n-1} \gamma_{i}\left(\pi\left(X_{2}\right)-\lambda\left(\pi_{2}\right) \mathrm{Id}\right)^{i}\right\} w_{1} \in H\left(\delta_{1}\right)
$$

and $\pi\left(X_{12}\right) \pi\left(X_{21}\right) \pi\left(X_{12}\right) w_{1} \in H\left(\delta_{1}\right), u=0$. Q.E.D.

3.7. Lemma. Assume there exists $\pi \in H\left(\pi_{1}, \pi_{2}\right)$ indecomposable and of length $n$. Assume furthermore that $\pi$ has a maximal subrepresentation $\pi^{\prime}$ such that $\pi / \pi^{\prime}$ is equivalent to $\pi_{1}$. Let $\gamma, \gamma_{2}, \ldots, \gamma_{n-2}$ be as in Theorem 3.3A. Then for each $\gamma_{n-1} \in \mathbf{C}$

$$
\bar{\pi}\left(X_{12}\right)\left\{\bar{\pi}\left(X_{2}\right)-\lambda\left(\pi_{2}\right) \mathrm{Id}-\gamma \bar{\pi}\left(X_{21} X_{12}\right)-\sum_{i=2}^{n-1} \gamma_{i}\left(\bar{\pi}\left(X_{2}\right)-\lambda\left(\pi_{2}\right) \mathrm{Id}\right)^{i}\right\}=0 .
$$

Proof. We have $\bar{\pi}\left(X_{2}\right)=\bar{\pi}^{\prime}\left(X_{2}\right)$. Thus

$$
\left(\bar{\pi}\left(X_{2}\right)-\lambda\left(\pi_{2}\right)\right)^{n-1}=0 . \quad \text { Q.E.D. }
$$

We continue by studying some special representations a bit more closely.

We say that $\pi$ satisfies condition (S), if $(\pi, H) \in H\left(\pi_{1}, \pi_{2}\right)$ is indecomposable and $\operatorname{dim} H\left(\delta_{1}\right)=\operatorname{dim} H\left(\delta_{2}\right)=2$.

3.8. LemMA. There exists a unique $\gamma$ such that for all $\pi$ satisfying condition $(S)$

$$
\bar{\pi}\left(X_{12}\right)\left\{\bar{\pi}\left(X_{2}\right)-\gamma \bar{\pi}\left(X_{21} X_{12}\right)-\lambda\left(\pi_{2}\right) \mathrm{Id}\right\}=0 .
$$

Proof. Let $\pi$ be a representation satisfying condition (S). Choose a basis $v_{1}, v_{2} \in H\left(\delta_{1}\right), u_{1}, u_{2}$ of $H\left(\delta_{2}\right)$ so that $\bar{\pi}\left(X_{12}\right) \bar{\pi}\left(X_{21}\right)$ and $\bar{\pi}\left(X_{21}\right) \bar{\pi}\left(X_{12}\right)$ are lower triangular matrices with one or zero in the lower left corner. 
Assume first $\bar{\pi}\left(X_{12}\right)\left\{\bar{\pi}\left(X_{2}\right)-\lambda\left(\pi_{2}\right) \mathrm{Id}\right\}=0$. So $\pi\left(X_{12}\right) u_{2}=0$. Since $\bar{\pi}\left(X_{21}\right) \bar{\pi}\left(X_{12}\right)$ is a lower triangular matrix

$$
\bar{\pi}\left(X_{12}\right) \bar{\pi}\left(X_{21}\right) \bar{\pi}\left(X_{12}\right)=0 .
$$

And thus any $\gamma \in \mathbf{C}$ satisfies the lemma.

Now assume

$$
\bar{\pi}\left(X_{12}\right) \pi\left(\left(X_{2}\right)-\lambda\left(\pi_{2}\right)\right) \neq 0
$$

Then we may assume

$$
\pi\left(X_{12}\right) u_{1}=a_{1} v_{1}, \quad \pi\left(X_{12}\right) u_{2}=v_{2}, \quad \pi\left(X_{21}\right) v_{1}=a_{3} v_{2}, \quad \pi\left(X_{21}\right) v_{2}=0
$$

with $a_{1} a_{3}=1$ or $0, a_{3}=1$ or 0 and

$$
\bar{\pi}\left(X_{2}\right)-\lambda\left(\pi_{2}\right) \mathrm{Id}=\left(\begin{array}{cc}
0 & 0 \\
\lambda_{1} & 0
\end{array}\right), \quad \lambda_{1} \in \mathbf{C} \backslash 0 .
$$

Since $X_{2} \in Z(g), \pi\left(X_{2}\right)$ is an intertwining operator for $\pi$, so is $\pi\left(X_{2}\right)-\lambda\left(\pi_{2}\right) \mathrm{Id}$. Their restrictions to $H\left(\delta_{1}\right) \oplus H\left(\delta_{2}\right)$ are intertwining operators for $\psi(\pi)$. So, in particular,

$$
\left(\pi\left(X_{2}\right)-\lambda\left(\pi_{2}\right) \mathrm{Id}\right)_{\mid H\left(\delta_{1}\right)}=\left(\begin{array}{cc}
0 & 0 \\
\lambda_{2} & 0
\end{array}\right), \quad \lambda_{2} \in \mathbf{C} \backslash 0,
$$

with $\lambda_{1}=\lambda_{2} a_{1} \neq 0$. So $\lambda_{2} \neq 0$ and $a_{1} \neq 0$. We will show next that $a_{3} \neq 0$. Assume $a_{3}=0$. Then $\left(\mathbf{C} v_{1}, 0,0,0,0\right)$ is an irreducible subspace of $\psi(\pi)$. Hence by Lemma 2.6 there exists an irreducible subrepresentation $\tilde{\pi}$ of $\pi$ such that $\psi(\tilde{\pi})=$ $\left(\mathbf{C} v_{1}, 0,0,0\right.$,). So $C v_{1}$ is invariant under $Z(g)$. On the other hand by (*) $\mathbf{C} v$ is not invariant. Hence $a_{3} \neq 0$.

Considering a representation equivalent to $\pi$, we may assume that $a_{1}=a_{3}=1$ and $\lambda_{1}=\lambda_{2}=\lambda$. To prove the lemma it suffices to show that we can choose the same constant $\lambda$ for all representations satisfying (S).

Let $\pi$ be such a representation. We first assume that $\psi(\pi)$ is in the prior form (we will refer to this form as standard form). Then $\pi$ has a maximal subrepresentation $\left(\pi^{\prime}, H^{\prime}\right)$ with

$$
\operatorname{dim} H^{\prime}\left(\delta_{1}\right)=2, \quad \operatorname{dim} H^{\prime}\left(\delta_{2}\right)=1 .
$$

But by 3.2(a) $\pi^{\prime}$ is uniquely determined by $\pi\left(X_{21}\right)_{\mid H^{\prime}\left(\delta_{1}\right)}$ and $\pi\left(X_{12}\right)_{\mid H^{\prime}\left(\delta_{2}\right)}$. Hence so is $\pi\left(X_{2}\right)_{\mid H\left(\delta_{1}\right)}$. Thus $\lambda_{1}=\lambda$ is uniquely determined by the representation $\pi^{\prime}$. So we put $\gamma=\lambda$. Q.E.D.

3.9. LemMA. There exists a unique $\mu \in \mathrm{C}$ such that for all $\pi$ satisfying condition $(S)$

$$
\left\{\bar{\pi}\left(X_{2}\right)-\lambda\left(\pi_{2}\right) \mathrm{Id}-\mu \bar{\pi}\left(X_{21}\right) \bar{\pi}\left(X_{12}\right)\right\} \bar{\pi}\left(X_{21}\right)=0 \text {. }
$$

Proof. The proof is analogous to the proof of Lemma 3.8.

3.10. LEMMA. Under the assumptions of Lemmas 3.8 and $3.9 \mu=\gamma$.

Proof. $\gamma$ and $\mu$ are both determined by $\pi\left(X_{2}\right)$, where $(\pi, H)$ is an indecomposable module with

$$
\operatorname{dim} H\left(\delta_{1}\right)=2 \text { and } \operatorname{dim} H\left(\delta_{2}\right)=1 .
$$

By Lemma 3.2(a), this determines $\gamma$ and $\mu$ uniquely. Q.E.D. 
REMARK. We established during the proof that if there exists a representation satisfying condition (S), then $Z(g)$ does not act semisimply on the representations considered in Lemma 3.2(a).

We say $\pi$ satisfies condition $\left(T_{n}\right)$ if

(a) $\pi \in H\left(\pi_{1}, \pi_{2}\right)$ is indecomposable; length $\pi=n>4$,

(b) $\pi$ has a unique irreducible subrepresentation $\tilde{\pi}_{1}$ and $\tilde{\pi}_{1} \cong \pi_{1}$.

(c) $\pi$ has a unique maximal submodule $\pi^{x}$ and $\pi / \pi^{x} \cong \pi_{2}$.

Let $\pi \in H\left(\pi_{1}, \pi_{2}\right)$. Since $Z(\mathrm{~g})$ acts nilpotently, there exists $m \in \mathbf{N}, m=m(\pi)$, such that

$$
\left(\pi\left(X_{2}\right)-\lambda\left(\pi_{2}\right) \mathrm{Id}\right)^{m}=0 \text { and }\left(\pi\left(X_{2}\right)-\lambda\left(\pi_{2}\right) \mathrm{Id}\right)^{m-1} \neq 0
$$

So

$$
\left(\pi\left(X_{2}\right)-\lambda\left(\pi_{2}\right) \mathrm{Id}\right)^{j}, \quad 1 \leqslant j \leqslant m,
$$

is a nontrivial intertwining operator. Let $0=\pi^{0} \subset \pi^{1} \subset \cdots \subset \pi^{m}=\pi$ be the filtration of $\pi$ defined by the kernels of $\left(\pi\left(X_{2}\right)-\lambda\left(\pi_{2}\right) \mathrm{Id}\right)^{m-j}, 0 \leqslant j \leqslant m$.

3.11. Lemma. Suppose $\pi$ satisfies condition $T_{n}$. Then $\pi / \pi^{m-1}$ is isomorphic to a nontrivial extension of $\pi_{1}$ by $\pi_{2}$.

Proof. (a) $\pi / \pi^{m-1}$ is equivalent to a subrepresentation of $\pi$ and so it has a unique subrepresentation equivalent to $\pi_{1}$.

(b) Since $\pi^{m-1} \subset \pi^{x}, \pi / \pi^{m-1}$ has a unique maximal submodule $\pi_{m}^{x}$ such that $\left(\pi / \pi^{m-1}\right) / \pi_{m}^{x}$ is isomorphic to $\pi_{2}$.

(c) Since $\pi / \pi^{m-1}$ is annihilated by $\pi\left(X_{2}-\lambda\left(\pi_{2}\right)\right), \pi\left(X_{2}\right)$ operates as a scalar on $\pi / \pi^{m-1}$.

To prove the lemma, it suffices to show that each representation with these properties is isomorphic to an extension of $\pi_{1}$ by $\pi_{2}$.

Let $U$ be such a representation. Write $\pi_{T}$ for a nontrivial extension of $\pi_{1}$ by $\pi_{2}$.

Assume first that $U$ has a subrepresentation $\tilde{U}$ isomorphic to a nontrivial extension of $\pi_{T}$ by $\pi_{1}$. Then $\tilde{U}$ is isomorphic to one of the representations of Lemma 3.2(a). Hence by property (b), $\tilde{U} \neq U$. By Lemma 3.2(c) the only nontrivial extension of $\tilde{U}$ by an irreducible representation in $H\left(\pi_{1}, \pi_{2}\right)$ satisfying (a) is isomorphic to a representation considered in Lemmas 3.8 and 3.9. Hence the proof of Lemma 3.8 implies that $X_{2}$ does not act as scalar on $\tilde{U}$ and thus on $U$.

Assume now that $U$ has a subrepresentation $\tilde{U}$ isomorphic to a nontrivial extension of $\pi_{T}$ by $\pi_{2}$. Thus by Lemma 3.2(b), $\tilde{U}$ has two irreducible subrepresentations. So we get a contradiction to (a).

On the other hand, (a) and (b) imply that $U$ has a subrepresentation isomorphic to $\pi_{T}$. So $U=\pi_{T}$. Q.E.D.

3.12. Lemma. Suppose $\pi$ satisfies condition $T_{n}$. Then $\pi^{1}$ is isomorphic to an extension of $\pi_{1}$ by $\pi_{2}$.

Proof. By Lemma 3.11, $\pi^{1}$ contains a subrepresentation isomorphic to $\pi_{T}$ and it has a unique subrepresentation isomorphic to $\pi_{1}$. Hence by Lemma 3.2(b) $\pi^{1}$ does not contain a subrepresentation isomorphic to an extension of $\pi_{T}$ by $\pi_{2}$. 
Assume $\pi^{1}$ contains a subrepresentation $\tilde{\pi}$ isomorphic to an extension of $\pi_{T}$ by $\pi_{1}$. Since $\pi \neq \tilde{\pi}$, by Lemma 3.2(c) $\pi$ contains a subrepresentation equivalent to one considered in Lemma 3.8. So $\pi\left(X_{2}\right)$ does not act semisimply on $\pi$ and hence $\tilde{\pi} \not \pi^{1}$. So $\pi^{1} \cong \pi_{T}$. Q.E.D.

3.13. Lemma. Suppose $\pi$ satisfies condition $T_{n}$. Then $\pi^{i} / \pi^{i-1}, i=1, \ldots, m$, is isomorphic to an extension of $\pi_{1}$ by $\pi_{2}$.

Proof. Consider $\pi / \pi^{m-2}$. This representation has at most length 4 , since $\pi^{m-1} / \pi^{m-2}$ is isomorphic to a subrepresentation of $\pi^{1} . \pi / \pi^{m-2}$ has a subrepresentation isomorphic to $\pi_{1}$ and is indecomposable.

Assume $\pi / \pi^{m-2}$ has length 3 . Then by Lemma $3.11, \pi^{m-1} / \pi^{m-2}$ is irreducible and isomorphic to $\pi_{1}$. Hence $\pi / \pi^{m-2}$ is isomorphic to a representation considered in Lemma 3.2(b) and therefore $\pi / \pi^{m-1} \cong \pi_{T}$. So $\pi$ has length 4 and is isomorphic to a representation considered in Lemma 3.8 and thus $\pi^{m-1} / \pi^{m-2} \simeq \pi_{T}$.

To complete the proof, repeat the argument for $\pi^{m-1}, \ldots, \pi^{1}$. Q.E.D.

REMARK. This implies, in particular, $\operatorname{dim} H\left(\delta_{1}\right)=\operatorname{dim} H\left(\delta_{2}\right)=m$ and thus $n=$ $2 m$.

3.14. Lemma. Suppose $\pi$ satisfies condition $T_{n}$. There exist $\gamma, \gamma_{2}, \ldots, \gamma_{n-1} \in \mathbf{C}$ so that

$$
\bar{\pi}\left(X_{12}\right)\left\{\bar{\pi}\left(X_{2}\right)-\lambda\left(\pi_{2}\right) \mathrm{Id}-\lambda \bar{\pi}\left(X_{21} X_{12}\right)-\sum_{i=2}^{n-1} \gamma_{i}\left(\bar{\pi}\left(X_{2}\right)-\lambda\left(\pi_{2}\right) \mathrm{Id}\right)^{i}\right\}=0
$$

Proof. Put $m=n / 2$. Choose a basis $v_{1}, \ldots, v_{m}$ of $H\left(\delta_{1}\right)$ and $w_{1}, \ldots, w_{m}$ of $H\left(\delta_{2}\right)$ according to the subspace structure. By Lemmas 3.8 and 3.13 and induction on $m$ we may assume

$$
\begin{aligned}
\bar{\pi}\left(X_{21}\right) v_{i} & =w_{i-1}, \quad i=2, \ldots, m-1, \\
\bar{\pi}\left(X_{21}\right) v_{m} & =0, \\
\bar{\pi}\left(X_{12}\right) w_{i} & =v_{i}, \quad i=2, \ldots, m,
\end{aligned}
$$

and

$$
\left(\bar{\pi}\left(X_{2}\right)-\lambda\left(\pi_{2}\right)\right) w_{i}=\gamma w_{i+1}+\beta_{1} w_{i+2}+\cdots+\beta_{m-i+1} w_{m}
$$

where $i=2, \ldots, m, \beta_{i} \in \mathbf{C}$, and $\gamma$ is as in Lemma 3.8; furthermore we may assume

$$
\left(\bar{\pi}\left(X_{2}\right)-\lambda\left(\pi_{2}\right) \mathrm{Id}\right) w_{m}=0,
$$

and

$$
\begin{gathered}
\bar{\pi}\left(X_{21}\right) v_{1}=w_{2}+\alpha_{1} w_{m}, \quad \bar{\pi}\left(X_{12}\right) w_{1}=v_{1}, \\
\left(\bar{\pi}\left(X_{2}\right)-\lambda\left(\pi_{2}\right) \mathrm{Id}\right) w_{1}=\gamma w_{2}+\sum_{j} \beta_{j} w_{j+1}+\alpha_{2} w_{2},
\end{gathered}
$$

where $\alpha_{1}, \alpha_{2} \in \mathbf{C}$. 
After a change of basis, we may assume that

$$
\begin{gathered}
\bar{\pi}\left(X_{21}\right) v_{i}=w_{i+1}, \quad \bar{\pi}\left(X_{12}\right) w_{i}=v_{i}, \quad i=1, \ldots, m, \\
\left(\bar{\pi}\left(X_{2}\right)-\lambda\left(\pi_{2}\right) \mathrm{Id}\right) w_{1}=\gamma w_{2}+\sum \beta_{j} w_{1+j} .
\end{gathered}
$$

Hence we get recursion relations for $\gamma_{2}, \ldots, \gamma_{2 m-1}$, which determine $\gamma_{2}, \ldots, \gamma_{m}$ uniquely, whereas $\gamma_{m+1}, \ldots, \gamma_{2 m-1}$ are arbitrary.

Since relations between $\bar{\pi}\left(X_{12}\right), \bar{\pi}\left(X_{21}\right), \bar{\pi}\left(X_{2}\right)$ depend only on the equivalence class of the representation, the lemma follows. Q.E.D.

REMARK. Each representation $\tilde{\pi}$ satisfying condition $T_{m}, m<n$, is equivalent to a subrepresentation of a representation $\pi$ satisfying condition $T_{n}$. So, if $\gamma, \gamma_{2}, \ldots, \gamma_{n-1}$ are the constants for $\pi$, then

$$
\overline{\tilde{\pi}}\left(X_{12}\right)\left\{\overline{\tilde{\pi}}\left(X_{2}\right)-\lambda\left(\pi_{2}\right) \mathrm{Id}-\gamma \overline{\tilde{\pi}}\left(X_{21} X_{12}\right)-\sum_{i=2}^{m-1} \gamma_{i}\left(\overline{\tilde{\pi}}\left(X_{2}\right)-\lambda\left(\pi_{2}\right) \mathrm{Id}\right)^{i}\right\}=0 .
$$

Proof of Theorem 3.3A. Suppose first $n$ is even. Suppose furthermore there exist $\pi \in H\left(\pi_{1}, \pi_{2}\right)$ indecomposable and satisfying condition $T_{n}$. Let, $\gamma, \gamma_{2}, \ldots, \gamma_{n-1}$ be as in Lemma 3.14. Each representation of length $n$ either satisfies condition $T_{n}$ or the assumptions of Lemmas 3.4-3.7. Thus it suffices to show that, for each representation $\tilde{\pi}$ of length $m<n, \gamma_{1}, \ldots, \gamma_{m-1}$ satisfy the conditions of the theorem. By applying Lemmas 3.4-3.7 again we see that it suffices to prove the theorem if $\pi$ satisfies condition $T_{m}, m<n$. In this case, it follows from the remark.

Now suppose $n$ odd and suppose there exists $\pi \in H\left(\pi_{1}, \pi_{2}\right)$ satisfying the condition $T_{n-1}$. Let $\gamma_{1}, \ldots, \gamma_{n}$ be the constants of Lemma 3.14. Each representation of length $n$ satisfies the conditions of Lemmas 3.4-3.7. Thus it suffices to show that for a representation $\pi$ of length $m<n, \gamma_{1}, \gamma_{2}, \ldots, \gamma_{m-1}$ satisfy the conditions of the theorem. Applying Lemmas 3.4-3.7 again, it suffices to prove the theorem if $\pi$ satisfies condition $T_{m}$ with $m<n-1$. In this case it follows from the remark.

Let $n_{0}$ be the largest integer such that there exists a representation satisfying condition $T_{n_{0}}$. Let $\gamma, \gamma_{2}, \ldots, \gamma_{n_{0}-1}$ be the constants of Lemma 3.14. Suppose $n>n_{0}$. Each representation of length $n$ satisfies the assumptions of Lemmas 3.4-3.7. Thus $\gamma_{1}, \ldots, \gamma_{n_{0}-1}, \gamma_{n_{0}}, \ldots, \gamma_{n-1}$ satisfy the conditions of the theorem for any choices of $\gamma_{n_{0}}, \ldots, \gamma_{n-1}$. Q.E.D.

Proof of Theorem 3.3B. Here we reduce the problem to computations for representations $\pi$ satisfying condition $Q_{n}$, i.e.,

(a) $\pi \in H\left(\pi_{1}, \pi_{2}\right)$ is indecomposable; length $\pi=n>4$,

(b) $\pi$ has a unique irreducible subrepresentation $\tilde{\pi}$ and $\tilde{\pi} \simeq \pi_{2}$,

(c) $\pi$ has a unique maximal submodule $\pi^{x}$ and $\pi / \pi^{x} \cong \pi_{1}$.

Then we continue as in the proof of Theorem 3.3A. Q.E.D.

3.15. Remark. Let $\pi \in H\left(\pi_{1}, \pi_{2}\right)$ be indecomposable. Suppose there exists a chain of subrepresentations $0=\pi^{0} \subset \pi^{1} \subset \cdots \subset \pi^{n}=\pi$ with $n$ odd such that

$$
\pi_{2 i} / \pi_{2 i-1} \cong \pi_{2}, \quad \pi_{2 i-1} / \pi_{2 i} \cong \pi_{1}
$$

and each subrepresentation is one of the representations $\pi^{i}$. 
Let $v_{1}, \ldots, v_{(n+1) / 2}$ and $w_{1}, \ldots, w_{(n-1) / 2}$ be a basis of $H\left(\delta_{1}\right)$ and $H\left(\delta_{2}\right)$ respectively, according to the subspace structure. We may assume

$$
\begin{gathered}
\pi\left(X_{21}\right) v_{i}=w_{i}, \quad i=1, \ldots,(n-1) / 2, \\
\pi\left(X_{21}\right) v_{(n+1) / 2}=0, \quad \pi\left(X_{12}\right) w_{i}=v_{i+1}, \\
\pi\left(X_{2}\right) w_{i}=\sum_{j} a_{i j} w_{i-j}+\lambda(\pi) w_{i} .
\end{gathered}
$$

Since the subrepresentation generated by $w_{1}$ satisfies condition $T_{n} a_{i 1}=\gamma, a_{i j}=\beta_{j}$. (Notation as in Lemma 3.14.) Hence all representations $\pi$ satisfying the above assumptions are equivalent.

3.16. Proposition. Let $n \in \mathbf{N}, \gamma, \gamma_{2}, \ldots, \gamma_{n-1}, \mu, \mu_{2}, \ldots, \mu_{n-1}$ be the constants of Theorems 3.3A and 3.3B respectively. We may choose $\mu=\gamma$ and $\mu_{i}=\gamma_{i}$.

Proof. Let $m_{0}$ be the largest integer such that there exist representations satisfying $T_{m_{0}}$ or $Q_{m_{0}}$. It suffices to prove the proposition for representations satisfying $T_{n}$ or $Q_{n}, n<m_{0}$, since we used these representations to calculate the constants.

Let $\pi$ be a representation satisfying $T_{n}, n \leqslant m_{0}, v_{1}, \ldots, v_{n / 2}, w_{1}, \ldots, w_{n / 2}$ be a basis of $H\left(\delta_{1}\right)$ and $H\left(\delta_{2}\right)$ according to the invariant subspace structure so that

$$
\begin{aligned}
\pi\left(X_{21}\right) v_{i} & =w_{i-1}, \quad i=1, \ldots, n / 2-1, \\
\pi\left(X_{21}\right) v_{n / 2} & =0, \\
\pi\left(X_{12}\right) w_{i} & =v_{i}, \quad i=1, \ldots, n / 2, \\
\pi\left(X_{2}\right) w_{i} & =\lambda\left(\pi_{2}\right) w_{i}+\gamma w_{i+1}+\sum \beta_{j} w_{i+j},
\end{aligned}
$$

and, since $\pi\left(X_{2}\right)$ is an intertwining operator,

$$
\pi\left(X_{2}\right) v_{i}=\lambda\left(\pi_{2}\right) v_{i}+\gamma v_{i+1}+\sum_{j} \beta_{j} v_{i+j}
$$

So $\gamma_{1}, \ldots, \gamma_{n / 2-1}$ are uniquely determined through the restriction of $\pi\left(X_{2}\right)$ to $H\left(\delta_{1}\right)$ and hence through the subrepresentation $\tilde{\pi}$ generated by $v_{1}$, and through the condition

$$
\left\{\pi\left(X_{2}-\lambda\left(X_{2}\right)\right)-\gamma \pi\left(X_{12} X_{21}\right)-\sum_{i=2}^{n / 2-1} \gamma_{i} \pi\left(X_{2}-\lambda\left(\pi_{2}\right)\right)^{i}\right\} \mid H\left(\delta_{1}\right)=0 .
$$

Let $\left(\pi^{\prime}, H^{\prime}\right)$ be a representation satisfying $Q_{n}, n \leqslant n_{0}, v_{1}^{\prime}, \ldots, v_{n / 2}^{\prime}, w_{1}^{\prime}, \ldots, w_{n / 2}^{\prime}$ be a basis of $H^{\prime}\left(\delta_{1}\right)$ and $H^{\prime}\left(\delta_{2}\right)$, respectively, according to the subspace structure such that

$$
\begin{aligned}
\pi^{\prime}\left(X_{21}\right) v_{i}^{\prime} & =w_{i}^{\prime}, \quad i=1, \ldots, n / 2, \\
\pi^{\prime}\left(X_{12}\right) w_{i}^{\prime} & =v_{i+1}^{\prime}, \quad i=1, \ldots, n / 2-1, \\
\pi^{\prime}\left(X_{12}\right) w_{n / 2}^{\prime} & =0, \\
\pi^{\prime}\left(X_{2}\right) w_{i}^{\prime} & =\lambda\left(\pi_{2}\right) w_{i}^{\prime}+\mu w_{i+1}^{\prime}+\sum_{j} \beta_{j}^{\prime} w_{i+j}^{\prime}
\end{aligned}
$$


and, since $\pi^{\prime}\left(X_{2}\right)$ is an intertwining operator,

$$
\pi^{\prime}\left(X_{2}\right) v_{i}^{\prime}=\lambda\left(\pi_{2}\right) v_{i}^{\prime}+\mu v_{i-1}^{\prime}+\sum_{j} \beta_{j}^{\prime} v_{i+j}^{\prime}
$$

Hence $\mu, \mu_{2}, \ldots, \mu_{n / 2-1}$ are uniquely determined through $\tilde{\pi}^{\prime}\left(X_{2}\right)$ where $\tilde{\pi}^{\prime}=$ $\pi^{\prime} / \pi_{2}$, and through the condition

$$
\left\{\tilde{\pi}^{\prime}\left(X_{2}-\lambda\left(\pi_{2}\right)\right)-\mu \tilde{\pi}^{\prime}\left(X_{12} X_{21}\right)-\sum_{i=2}^{n / 2-1} \mu_{i} \tilde{\pi}^{\prime}\left(X_{2}-\left(\pi_{2}\right)\right)^{i}\right\} \mid H\left(\delta_{1}\right)=0 .
$$

By Lemma 3.15, $\tilde{\pi}$ and $\tilde{\pi}^{\prime}$ are equivalent. Hence $\mu=\gamma, \mu_{i}=\gamma_{i}, i=2, \ldots, n / 2-$ 1. Since $\mu_{i}, i=n / 2, \ldots, n-1$ and $\gamma_{i}$ for $i=n / 2, \ldots, n-1$ are arbitrary, the proposition follows. Q.E.D.

We now define a new functor $\psi_{0}$. Let $\pi \in H\left(\pi_{1}, \pi_{2}\right)$. Assume length $\pi=n$. Let $\gamma, \gamma_{2}, \ldots, \gamma_{n-1}$ be the constants determined by Remark 3.15. Define

$$
\begin{aligned}
\psi_{0}(\pi)=\left(H\left(\delta_{1}\right), H\left(\delta_{2}\right)\right. & \bar{\pi}\left(X_{21}\right), \bar{\pi}\left(X_{12}\right), \bar{\pi}\left(X_{2}\right) \\
& \left.-\gamma \bar{\pi}\left(X_{21} X_{12}\right)-\lambda\left(\pi_{2}\right) \mathrm{Id}-\sum_{i=2}^{n-1} \gamma_{i}\left(\bar{\pi}\left(X_{2}\right)-\lambda\left(\pi_{2}\right) \mathrm{Id}\right)^{i}\right) .
\end{aligned}
$$

Note that if $\pi=\pi_{1} \oplus \pi_{2}$, then $\psi_{0}(\pi)=\psi_{0}\left(\pi_{1}\right) \oplus \psi_{0}\left(\pi_{2}\right)$.

Let $\mathfrak{X}$ be the category of all objects consisting of two vector spaces $V_{1}, V_{2}$ and mappings

$$
d^{+}: V_{1} \rightarrow V_{2}, \quad d^{-}: V_{2} \rightarrow V_{1}, \quad \delta: V_{2} \rightarrow V_{2}
$$

with the properties

(a) $\delta, d^{+} d^{-}$, and $d^{-} d^{+}$are nilpotent,

(b) $\delta d^{-}=d^{+} \delta=0$.

$\mathfrak{X}$ is a subcategory of the category of all representations of the diagram $D$.

3.17. THEOREM. $\psi_{0}$ is a monomorphism of $H\left(\pi_{1}, \pi_{2}\right)$ into a subcategory of $\mathfrak{X}$. Furthermore, $\psi_{0}$ maps indecomposable representations in $H\left(\pi_{1}, \pi_{2}\right)$ into indecomposable objects in $\mathfrak{X}$. Put

Proof. For $n \in \mathbf{N}$ let $\gamma, \gamma_{2}, \ldots, \gamma_{n-1}$ be the constants satisfying Theorem 3.3A.

$$
{ }_{n} X_{2}=X-\lambda\left(\pi_{2}\right)-\sum_{i=2}^{n-1} \gamma_{i}\left(X_{2}-\lambda\left(\pi_{2}\right)\right)^{i}-\gamma \pi\left(X_{21} X_{12}\right)
$$

and define the functor $\psi_{n}$ by

$$
\psi_{n}(\pi)=\left(H\left(\delta_{1}\right), H\left(\delta_{2}\right), \bar{\pi}\left(X_{21}\right), \bar{\pi}\left(X_{12}\right), \bar{\pi}\left({ }_{n} X_{2}\right)\right) \text { for } \pi \in H\left(\pi_{1}, \pi_{2}\right) .
$$

By the remark which follows it, Theorem 2.11 is valid if we replace $X_{2}$ by ${ }_{n} X_{2}$ and $\psi$ by $\psi_{n}$.

Since $\psi_{n}(\pi)=\psi_{0}(\pi)$ for all representations $\pi$ of length shorter than $n, \psi_{0}$ is a monomorphism and maps indecomposable representations into indecomposable representations. By Theorems 3.3A, 3.3B, and Proposition 3.16, the image of $\psi_{0}$ lies in $\mathfrak{X}$ Q.E.D. 
In $H\left(\pi_{1}, \pi_{2}\right)$ we have the following operations to construct new modules:

(A) Let $\left(\pi^{1}, H^{1}\right),\left(\pi^{2}, H^{2}\right) \in H\left(\pi_{1}, \pi_{2}\right)$ and $\left(\tilde{\pi}^{1}, \tilde{H}^{1}\right) \subset\left(\pi^{1}, H^{1}\right),\left(\tilde{\pi}^{2}, \tilde{H}^{2}\right) \subset$ $\left(\pi^{2}, H^{2}\right)$ be isomorphic submodules and let $\alpha: \tilde{\pi}^{1} \rightarrow \tilde{\pi}^{2}$ be a given isomorphism. The module

$$
\pi(\alpha)=\left(\pi^{1} \oplus \pi^{2}\right) / \pi
$$

where $\pi$ is the submodule consisting of the pairs $(h, \alpha(h)) h \in \tilde{H}^{1}$ is called the glueing of $\pi^{1}$ and $\pi^{2}$ over the submodules $\tilde{\pi}^{1}$ and $\tilde{\pi}^{2}$. We call this "operation $G_{1}$ ".

(B) Let $\left(\pi^{1}, H^{1}\right),\left(\pi^{2}, H^{2}\right) \in H\left(\pi_{1}, \pi_{2}\right)$ and let $\left(\tilde{\pi}^{1}, \tilde{H}^{1}\right) \subset\left(\pi^{1}, H^{1}\right),\left(\tilde{\pi}^{2}, \tilde{H}^{2}\right) \subset$ $\left(\pi^{2}, H^{2}\right)$ be submodules such that we have an isomorphism $\alpha: \pi^{1} / \tilde{\pi}^{1} \rightarrow \pi^{2} / \tilde{\pi}^{2}$. The submodule $\tilde{\pi}(\alpha) \subset \pi_{1} \oplus \pi_{2}$ consisting of all pairs $\left(h_{1}, h_{2}\right), h_{1} \in H^{1}, h_{2} \in H^{2}$ such that $\alpha \bar{h}_{1}=\bar{h}_{2}$ where $\bar{h}_{i}=h_{i} \bmod \tilde{H}^{i}$, will be called the glueing of $H^{1}$ and $H^{2}$ over the factor modules $\pi^{i} / \tilde{\pi}^{i}, i=1,2$. We refer to this as "operation $G_{2}$ ".

(C) Let $(\pi, H) \in H\left(\pi_{1}, \pi_{2}\right)$, and let $\left(\pi^{1}, H^{1}\right),\left(\pi^{2}, H^{2}\right), H^{1} \neq H^{2}$ be isomorphic submodules of $(\pi, H)$. Fix the isomorphism $\alpha: \pi^{1} \rightarrow \pi^{2}$. The module

$$
\pi^{m}(\lambda, \alpha)=\left(\bigoplus_{i=1}^{m} \pi\right) / \pi^{\lambda}
$$

where $\pi^{\lambda}$ is the submodule consisting of

$$
\left(\alpha h_{1}-\lambda h_{1}, \alpha h_{2}-\lambda h_{2}-h_{1}, \ldots, \alpha h_{m}-\lambda h_{m}-h_{m-1}\right), \quad h_{i} \in H^{1}, \lambda \in \mathbf{C} \backslash 0,
$$

is called a polymerisation of $m$ copies of the module $(\pi, H)$. We refer to this as "operation $P_{m}$ ".

REMARK. We have

$$
\begin{gathered}
\pi(\alpha)=\pi(\lambda \alpha), \quad \pi(\alpha)=\pi(\lambda \alpha), \quad \lambda \in \mathbf{C} \backslash 0, \\
\pi^{m}(\lambda, \alpha)=\pi^{m}\left(\lambda^{1} \lambda, \lambda^{1} \alpha\right), \quad \lambda^{1} \in \mathbf{C} \backslash 0 .
\end{gathered}
$$

Definition. We call an indecomposable representation $(\pi, H) \in H\left(\pi_{1}, \pi_{2}\right)$ elementary if one of the following conditions is satisfied.

Suppose $0=\left(\pi^{0}, H^{0}\right) \subset\left(\pi^{1}, H^{1}\right) \subset \cdots \subset\left(\pi^{n}, H^{n}\right)=(\pi, H)$ is a maximal nontrivial chain of submodules. Then either

(a) for all $i \in\{0, \ldots, n / 2\}, \pi^{i+1} / \pi^{i} \cong \pi_{2}$ or

(b) for all $i \in\{0, \ldots, n / 2\}, \pi^{2 i} / \pi^{2 i-1} \cong \pi_{l}$ and $\pi^{2 i+1} / \pi^{2 i} \cong \pi_{k}$ where $l \neq k$, and if $\operatorname{dim} H\left(\delta_{2}\right)>\operatorname{dim} H\left(\delta_{1}\right)$, then

$$
\bar{\pi}\left(X_{2}-\lambda\left(\pi_{2}\right)\right)=\gamma \bar{\pi}\left(X_{21} X_{12}\right)-\sum_{i=2}^{(n-1) / 2} \gamma_{i}\left(\bar{\pi}\left(X_{2}-\lambda\left(\pi_{2}\right)\right)\right)^{i}
$$

for suitable $\gamma, \gamma_{1}, \ldots, \gamma_{(n-1) / 2}$.

3.18. THEOREM. Each representation in $H\left(\pi_{1}, \pi_{2}\right)$ can be constructed out of elementary representations using the operations $G_{1}, G_{2}, P_{m}, m \in \mathbf{N}$.

Proof. We can define elementary representations and the operations $G_{1}, G_{2}, P_{m}$, $m \in N$ in the abelian category $\mathfrak{X}$ as well. By [6], Theorem 3.18 is true in $\mathfrak{X}$. Since $\psi_{0}$ intertwines the operations $G_{1}, G_{2}$ and $P_{m}$ in $H\left(\pi_{1}, \pi_{2}\right)$ and in $\mathfrak{X}$ the theorem follows. Q.E.D. 
Remark. Theorem 3.18 was first proved by Gelfand and Ponomarev [7] for indecomposable representations of $\mathrm{Sl}(2, \mathrm{C})$.

4. Extensions of two representations: the general theory. Let $G=K A N$ be an Iwasawa decomposition of $G, P=M A N$ a minimal parabolic subgroup of $G, \Sigma^{+}$ the set of positive roots determined through $N$ and $\rho=\frac{1}{2} \Sigma_{\alpha \in \Sigma^{+}} \alpha$.

For an admissible $(\mathrm{g}, K)$ module $(\pi, H)$ we define $H_{(\mathrm{n})}=\left\{u \mid u=\pi(x) u^{\prime}\right.$ where $u^{\prime} \in H, X \in \mathfrak{n}$ ]. By [2], $H_{\mathfrak{n}}=H / H_{(\mathfrak{n})}$ is a finite-dimensional $U(\mathfrak{p})$-module and the representation of $\mathfrak{p}$ on $H_{\mathrm{n}}$ gives rise to a representation $\pi_{\mathrm{n}}$ of $P$ on $H_{\mathrm{n}}$.

Thus for each finite-dimensional representation $\tau$ of $P=M A N$ with $\tau($ man $)=$ $\tau(m a)$ we have the Frobenius reciprocity

$$
\operatorname{Hom}_{(\mathrm{g}, K)}\left(\pi, \operatorname{ind}_{\rho}^{G} \tau\right)=\operatorname{Hom}_{P}\left(\pi_{\mathrm{n}}, \tau \otimes \rho\right) .
$$

Here we identify $\rho$ and the function $\rho($ man $)=a^{\rho}=e^{\rho(\log a)}$.

Let $\left(\pi^{1}, H^{1}\right),\left(\pi^{2}, H^{2}\right)$ be irreducible admissible $(g, K)$-modules. Suppose $\pi$ is an extension of $\pi^{1}$ by $\pi^{2}$. Thus there exists a short exact sequence

$$
0 \rightarrow\left(\pi^{1}, H^{1}\right) \rightarrow(\pi, H) \rightarrow\left(\pi^{2}, H^{2}\right) \rightarrow 0
$$

and therefore

$$
0 \rightarrow H_{(\mathfrak{n})} \cap H^{1} \rightarrow H_{(\mathfrak{n})} \rightarrow H_{(\mathfrak{n})} / H_{(\mathfrak{n})} \cap H^{1} \rightarrow 0
$$

Hence

$$
0 \rightarrow H^{1} / H_{(\mathfrak{n})} \cap H^{1} \rightarrow H / H_{(\mathfrak{n})} \rightarrow H^{2} /\left(H_{(\mathfrak{n})} / H_{(\mathfrak{n})} \cap H^{1}\right) \rightarrow 0
$$

is a short exact sequence of $P$-modules. But

$$
H^{2} /\left(H_{(\mathfrak{n})} / H_{(\mathfrak{n})} \cap H^{1}\right) \simeq H_{\mathfrak{n}}^{2}
$$

and hence

$$
0 \rightarrow H^{1} / H_{(\mathfrak{n})} \cap H^{1} \rightarrow H / H_{(\mathfrak{n})} \rightarrow H_{\mathfrak{n}}^{2} \rightarrow 0 .
$$

Now let $(\pi, H)$ be an extension of $\pi^{2}$ by $\pi^{1}$. Then by the same argument there exists a short exact sequence

$$
0 \rightarrow H^{2} / H_{(\mathfrak{n})} \cap H^{2} \rightarrow H / H_{(\mathfrak{n})} \rightarrow H_{\mathfrak{n}}^{1} \rightarrow 0 .
$$

4.1. LemMA. Either $H^{1} / H_{(\mathfrak{n})} \cap H^{1}$ or $H^{2} / H_{(\mathfrak{n})} \cap H^{2}$ is nontrivial.

The proof of Lemma 4.1 uses some results about leading exponents.

Let $(\pi, H)$ be an admissible $(g, K)$-module, $\mathrm{C}_{\mathrm{a}}^{+}$be the positive Weyl chamber of a (as determined by the choice of the $N$ ), $u \in H$ and $\tilde{u}$ a $K$-finite vector in the dual of $H$. Then by [2], the coefficient $\langle\tilde{u}, \pi(a) u\rangle, \log a \in-\mathcal{C}_{\mathrm{a}}^{+}$, has the expansion

$$
\langle\tilde{u}, \pi(a) u\rangle=\sum_{\lambda \in \xi(\pi, u, \tilde{u})} P_{\lambda, u, \tilde{u}}(\log a) a^{\lambda} .
$$

Here $\xi(\pi, u, \tilde{u})$ is a countable set in $a^{\prime}$ and $p_{\lambda, u, \tilde{u}}$ is a polynomial on a for fixed $\lambda, u$, u.

We say $\mu$ is an exponent of $\langle\tilde{u}, \pi(g) u\rangle$ if $a^{\mu}$ has a nonzero coefficient in the previous expansion. 
Denote by $\xi(\pi)$ the union of all exponents of all $K$-finite matrix coefficients of $\pi$. Let $\lambda, \mu \in \mathfrak{a}^{\prime}$; then we write $\mu<\lambda$ if $\lambda-\mu=\Sigma_{\alpha_{i} \in \Sigma^{+}} n_{i} \alpha, n_{i} \in \mathbf{N} \backslash\{0\}$ and $\Sigma^{+}$the set of positive roots in $\mathfrak{a}^{\prime}$.

Finally, call the minimal elements $\xi^{0}(\pi)$ of $\xi(\pi)$ with respect to this ordering relation the leading exponents of $\pi$.

If $\pi_{0}$ is a composition factor of $\pi$, then obviously $\xi^{0}\left(\pi_{0}\right) \subset \xi(\pi)$.

Now define $\psi_{(\tilde{u}, u)}(a)=\Sigma_{\lambda \in \xi^{0}(\pi)} P_{\mu, u, \tilde{u}}(\log a) a^{\lambda}$. For $\log a \in-\mathcal{C}_{a}^{+}, \psi_{(,,)}(a)$ is a bilinear map $H^{\prime} \times H \rightarrow \mathrm{C}$ (here $H^{\prime}$ denotes the $K$-finite dual of $H$ ) [12].

4.2. Lemma. There exists an $a \in \exp \left(-\mathrm{C}_{\mathrm{a}}^{+}\right)$such that $\psi_{(,,)}(a)$ is nontrivial.

Proof. Choose $u, \tilde{u}$ so that at least one of the leading exponents of $\pi$ is an exponent of $\langle\tilde{u}, \pi(g) u\rangle$. Then $\psi_{(\tilde{u}, u)}()$ is a finite sum of distinct exponential functions with polynomial coefficients and thus does not vanish on an open subset of $\mathbf{R}^{n}$. Hence we can choose a $a \in \exp \left(-C_{a}^{+}\right)$such that $\psi_{(u, u)}(a) \neq 0$. Q.E.D.

Fix $a \in \exp \left(-C_{\mathrm{a}}^{+}\right)$so that $\psi_{(,)}(a)$ is nontrivial and write $\psi($,$) for the form$ $\psi_{(,,)}(a)$.

4.3. Lemma. $\psi(\tilde{u}, \pi(X) u)=0$ for all $X \in \mathfrak{n}, \tilde{u} \in H^{\prime}$.

Proof. This is an immediate consequence of [2]. Q.E.D.

Now return to the proof of Lemma 4.1. If $\xi^{0}(\pi) \subset \xi\left(\pi_{2}\right)$ and $\xi^{0}\left(\pi_{1}\right) \neq \xi^{0}\left(\pi_{2}\right)$, let $(\pi, H)$ to be an extension of $\pi_{2}$ by $\pi_{1}$. If $\xi^{0}\left(\pi_{2}\right) \subset \xi\left(\pi_{1}\right)$ and $\xi^{0}\left(\pi_{1}\right) \neq \xi^{0}\left(\pi_{2}\right)$, let $(\pi, H)$ to be an extension of $\pi_{2}$ by $\pi_{1}$. In the remaining case, choose $\pi$ to be either one. We denote by $(\check{\pi}, \check{H})$ the unique nontrivial subrepresentation of $\pi$ on the space $\check{H}$.

For each $\tilde{v} \in H^{\prime}, \psi(\tilde{v}$,$) is a linear functional on H$, whose restriction to $\check{H} \cap H_{(\mathfrak{n})}$ is not identical to zero.

To prove Lemma 4.1 , by Lemma 4.3 it suffices to show that $\psi(\tilde{v}$,$) is not$ identically zero on $\check{H}$ for all $\tilde{v} \in H^{\prime}$. Since $\xi^{0}(\pi) \cap \xi^{0}(\check{\pi}) \neq 0$ by construction of $\pi$, and since each exponent of $\check{\pi}$ is also an exponent of $\pi$, the consideration in the proof of Lemma 4.2 shows that we can find $\tilde{v}$ and an $a \in A$ so that $\psi_{(\tilde{v}, v)}(a) \neq 0$ for some $v$ in $\check{H}$. Q.E.D.

Consider a finite-dimensional, not necessarily irreducible representation $\tau$ of $P / N$ and lift it to a representation of $P$. The representation ind ${ }_{P}^{G} \tau$ is called Jordan representation. Here we define induction in such a way that $G$ acts on the left and the induced representation is unitary if $\tau$ is unitary. If $\tau$ is irreducible, ind ${ }_{P}^{G} \tau$ is a principal series representation.

4.4. Proposition. Let $\pi_{1}, \pi_{2}$ be irreducible representations of $G$ and suppose $\pi$ is defined as is the proof of Lemma 4.1. Then $\pi$ is equivalent to a subrepresentation of $a$ Jordan representation.

Proof. This follows from Lemma 4.1 and Frobenius reciprocity. Q.E.D.

We close this section with some remarks about "leading exponent embeddings". Let $\pi$ be a representation, $\mu$ a leading exponent of $\pi$.W. Casselman observed that $\mu$ 
is a weight of $A$ on $H_{\mathrm{n}}$. Hence there exists a representation $\tau$ of $M$ so that

$$
\operatorname{Hom}_{U(\mathrm{~g})}\left(\pi, \operatorname{ind}_{P}^{G} \tau \otimes \mu\right)=0 .
$$

Here we consider $\tau \otimes \mu$ as a representation of $P$ which is trivial on $N$.

Now suppose $\pi_{1}, \pi_{2}$ are representations, $\pi_{1}$ is irreducible and $\xi^{0}\left(\pi_{1}\right) \subset \xi\left(\pi_{2}\right)$. Suppose $\pi$ is a nontrivial extension of $\pi_{1}$ by $\pi_{2}$ and $\pi_{1}$ is the socle of $\pi$. Let $\mu \in \xi^{0}\left(\pi_{1}\right), \mu \notin \xi\left(\pi_{2}\right)$ and consider the embedding in a principal series representation defined by $\mu$. Since $\pi_{1}$ is the socle of $\pi$, the embedding is either an isomorphism or its kernel contains $\pi_{1}$. But this latter case is impossible since this embedding is in particular an embedding of $\pi_{1}$. Thus we proved

4.5. COROLlaRY. Under the prior assumptions, $\pi$ is isomorphic to a subrepresentation of a principal series representation.

5. Extensions of finite-dimensional representations by infinite-dimensional representations. Let $\pi_{1}$ be a finite-dimensional irreducible representation of $G$. In this section we show that

$$
\operatorname{dim} \operatorname{Ext}_{U(g)}^{1}\left(\pi_{1}, \pi_{2}\right)<1
$$

for all irreducible representations $\pi_{2}$.

We start with some technical lemmas.

5.1. Lemma. Let $P=M A N$ be a parabolic subgroup of $G, \tau$ a finite-dimensional indecomposable representation of $M A$. Then $\tau \cong \tau_{M} \otimes \chi$, where $\tau_{M}$ is an irreducible representation of $M$ and $\chi$ an indecomposable representation of $A$.

Proof. $M A$ is a direct product of a vector group $A$ with a reductive group $M$. There exists a covering $\tilde{M}^{0}$ of $M^{0}$ such that $\tilde{M}^{0} \cong M_{s} \times M_{c}$ where $M_{s}$ is a semisimple group and $M_{c}$ is a compact group. Thus all finite-dimensional representations of $\tilde{M}^{0}$, and hence of $M$, are semisimple, and thus all indecomposable representations of $M A$ are tensor products of irreducible representations of $M$ with indecomposable representations of $A$. Q.E.D.

5.2. LemMA. Let $\chi$ be a character of the additive group of $\mathbf{R}$. Then $\operatorname{dim} \operatorname{Ext}_{\mathbf{R}}^{1}(\chi, \chi)$ $=1$.

Proof. Each twofold self-extension of $\chi$ is equivalent to the function

$$
\chi: a \rightarrow\left(\begin{array}{cc}
\chi(a) & (1-a) \chi(a) \\
0 & \chi(a)
\end{array}\right) \text { Q.E.D. }
$$

Let $G=K A N$ be the Iwasawa decomposition of $G, \Sigma^{+}$the set of positive roots of $(\mathfrak{g}, \mathfrak{a})$ determined by the choice of $N$.

5.3. Lemma. Suppose $\chi_{0}$ is a two-dimensional indecomposable representation of $A$ with a subrepresentation equivalent to $\chi$. Then $\chi_{0}$ is a two-fold self-extension of $\chi$.

The proof is left to the reader.

5.4. Lemma. Let $\pi: G \rightarrow$ Aut $H$ be a finite-dimensional irreducible representation of $G . \pi$ has a unique leading exponent which is contained in the closure of the negative Weyl chamber. 
Proof. The leading exponent of $\pi$ is the lowest weight (with respect to $\Sigma^{+}$). Q.E.D.

In particular, the leading exponent of a finite-dimensional representation $\pi: G \rightarrow$ Aut $H$ is the only weight of a on $H_{\mathfrak{n}}$. So by Frobenius reciprocity and [10] there is a unique principal series representation $U(\pi)$ with subrepresentation $\pi$.

5.5. Proposition. Let $\pi_{1}$ be a finite-dimensional irreducible representation of $G$. Each nontrivial extension $\pi$ of $\pi_{1}$ by an irreducible representation $\pi_{2}$ is equivalent to a subrepresentation of the principal series representation $U\left(\pi_{1}\right)$.

Proof. Let $\mathfrak{h}_{\mathbf{c}}$ be a Cartan subalgebra of $\mathfrak{g}_{\mathbf{C}}$. We may assume $\mathfrak{g} \cap \mathfrak{h}_{\mathbf{c}}=\mathfrak{t} \oplus \mathfrak{a}$ where $\mathfrak{t}=\mathfrak{h}_{\mathbf{C}} \cap \mathfrak{f}$. For $\lambda \in \mathfrak{h}^{\prime}$ we write $\lambda=\left(\lambda_{1}, \lambda_{2}\right)$ with $\lambda_{1} \in \mathfrak{t}^{\prime}, \lambda_{2} \in \mathfrak{a}^{\prime}$.

Let $\Delta^{+}$be a set of positive roots of $\left(\mathfrak{h}_{\mathbf{C}}, \mathfrak{g}_{\mathbf{C}}\right)$ compatible with $\Sigma^{+}$. Put $\rho$ $=\frac{1}{2} \sum_{\alpha \in \Delta^{+}} \alpha$.

Let $\gamma \in e^{+} \subset \mathfrak{h}^{\prime}$ be the infinitesimal character of $\pi_{1}$. By Lemma $1.2 \gamma$ is also the infinitesimal character of $\pi_{2}$. All translates of $\gamma-\rho$ under $W_{C}$ are weights of $\pi_{1}$. There exists a $\gamma \in\left\{w(\gamma-\rho), w \in W_{\mathbf{C}}\right\}$ such that $\gamma_{2}$ is the leading exponent of $\pi_{1}$. Furthermore $\left\langle\gamma_{2}, \gamma_{2}\right\rangle \geqslant\left\langle\gamma_{2}^{\prime}, \gamma_{2}^{\prime}\right\rangle$ for all $\gamma^{\prime} \in\left\{w(\gamma-\rho), w \in W_{\mathbf{C}}\right\}$.

By [10] the leading exponent of a principal series representation is contained in the negative Weyl chamber of $a^{\prime}$ and in particular the leading exponent of $U\left(\pi_{1}\right)$ is equal to $\gamma_{2}$. Furthermore all composition factors of $U\left(\pi_{1}\right)$ with exception of $\pi_{1}$ have leading exponents of length smaller than $\gamma_{2}$.

By Proposition $4.4 \pi$ is equivalent to a subrepresentation of a Jordan representation ind ${ }_{P}^{G} \tau$, where $\tau$ is a representation of $M A N$, indecomposable and of length at most 2. By Lemma 5.1 $\tau=\tau_{M} \otimes \chi$, where $\chi$ is a two-dimensional indecomposable representation of $A$. Since $U\left(\pi_{1}\right)$ is a subrepresentation of $\operatorname{ind}_{P}^{G} \tau$, Lemma 5.4 implies that $\chi$ is a two-dimensional extension of $\gamma_{2}$. Hence $\operatorname{ind}_{P}^{G} \tau$ is a two fold self-extension of $U\left(\pi_{1}\right)$ and therefore all composition factors of ind ${ }_{P}^{G} \tau$ with leading exponent $\gamma_{2}$ are equivalent to $\pi_{1}$.

Now the proposition follows from 4.6. Q.E.D.

Recall some definitions and results of [10], [15], [18]. Let $P=M_{P} A_{P} N_{P}$ be a parabolic subgroup of $G, \Sigma_{P}$ the roots of $\left(\mathfrak{g}, \mathfrak{a}_{p}\right)$ and $\Sigma_{p}$ the set of positive roots determined through $N_{p}$. We identify $\chi \in a_{p}^{\prime}$ with the function $\chi($ an $)=a^{\chi}=$ $e^{\chi(\log a)}, a \in A, n \in N$. Let $\tau$ be a tempered representation of $M_{p}, \chi \in a_{p}^{\prime}$. The representation $U(P, \tau, \chi)=\operatorname{ind}_{P}^{G} \tau \otimes \chi$ is called a generalized principal series representation. If $P$ is minimal, generalized principal series representations and principal series representations coincide.

Assume $\operatorname{Re} \chi$ has a strictly negative inner product with all roots $\alpha \in \Sigma_{P}$. Then $U(P, \tau, \chi)$ has a unique irreducible subrepresentation $\overline{U(P, \tau, \chi)}$, the Langlands subquotient of $U(P, \tau, \chi)$ [11]. Each irreducible representation $\pi$ of $G$ is equivalent to a representation $\overline{U(P, \tau, \chi)}$ for some $P, \tau, \chi$. We call $P, \tau, \chi$ the Langlands parameter of $\pi$ if $\pi=\overline{U(P, \tau, \chi)}$. Furthermore, if $P$ is cuspidal and $\chi$ is singular with respect to some root, then

$$
U(P, \tau, \chi)=\bigoplus_{i \in I} U\left(P_{i}, \tau_{i}, \chi_{i}\right)
$$


Here $I$ is finite and each $U\left(P_{i}, \tau_{i}, \chi_{i}\right)$ is a generalized principal series representation, $P_{i}=M_{i} A_{i} N_{i} \supset P$ and $\operatorname{Re} \chi$ is nonsingular with respect to all roots of $a_{i}$ in $n_{i}$.

Let $\pi$ be a Harish-Chandra module. We define

$$
\begin{aligned}
& P_{\gamma}(\pi)=\{v \in \pi \mid \text { there exists a positive integer } n \text { such that } \\
& \left.\qquad(z-\psi(z)(\gamma))^{n} v=0 \text { for all } z \in Z(g)\right\} .
\end{aligned}
$$

Then $\pi=\bigoplus_{\gamma \in \bar{e}} P_{\gamma}(\pi)$ is a finite sum.

Let $\pi_{\delta}$ be a finite-dimensional irreducible $\mathfrak{g}$-module with highest weight $\delta \in \bar{\complement}$ and $\pi_{\delta}^{*}$ its contragradient. Since $\pi \otimes \pi_{\delta}$ and $\pi \otimes \pi_{\delta}^{*}$ are Harish-Chandra modules for a finite covering group of $G$ we can define

$$
\psi_{\gamma}^{\gamma+\delta}(\pi)=P_{\gamma+\delta}\left(P_{\gamma}(\pi) \otimes \pi_{\delta}\right)
$$

and

$$
\phi_{\gamma-\delta}^{\gamma}(\pi)=P_{\gamma-\delta}\left(P_{\gamma}(\pi) \otimes \pi_{\delta}^{*}\right) .
$$

The stabilizer of $\gamma$ in the Weyl group $W_{\mathrm{C}}$ is denoted by $W_{\gamma}$.

5.6. TheOREM [15], [18]. (a) If $W_{\gamma}=W_{\gamma-\delta}, \psi_{\gamma-\delta}^{\gamma}$ and $\phi_{\gamma-\delta}^{\gamma}$ are monomorphisms, $\psi_{\gamma-\delta}^{\gamma} \phi_{\gamma-\delta}^{\gamma} \pi=\pi$ for any representation $\pi$ with infinitesimal character $\gamma$. In particular, the image of a generalized principal series representation is a generalized principal series representation.

(b) If $W_{\gamma} \subset W_{\gamma-\delta}$ and $\left|W_{\gamma}\right|=1$, then the image of an irreducible Harish-Chandra module under $\phi_{\gamma-\delta}^{\gamma}$ is either irreducible or zero and the image of a generalized principal series representation is zero or a generalized principal series representation.

(c) If $\pi$ is the Langlands subquotient of a generalized principal series representation $U$ and $\phi_{\gamma-\delta}^{\gamma} \pi \neq 0$, then $\phi_{\gamma-\delta}^{\gamma} \pi$ is the Langlands subquotient of $\phi_{\gamma-\delta}^{\gamma} U$.

Suppose $\pi_{1}$ is a finite-dimensional irreducible representation with infinitesimal character $\gamma \in e^{+}$(with respect to $\Sigma^{+}\left(\mathfrak{g}_{\mathbf{C}}, \mathfrak{h}_{\mathbf{C}}\right)$ ). For each simple root $\beta \in$ $\Sigma^{+}\left(\mathfrak{g}_{\mathbf{C}}, \mathfrak{h}_{\mathbf{C}}\right)$ let $\delta(\beta)$ be the fundamental weight with $\langle\delta(\beta), \beta\rangle=1$. Put $\gamma(\beta)=$ $(2\langle\gamma, \beta\rangle /\langle\beta, \beta\rangle) \delta(\beta)$. There exists a finite-dimensional representation of the universal covering group with highest weight $\gamma(\beta)$. In [15] it was shown that for each infinite-dimensional representation $\pi_{2}$ with infinitesimal character $\gamma$ there exists a simple root $\beta \in \Sigma^{+}\left(\mathfrak{g}_{\mathbf{C}}, \mathfrak{h}_{\mathbf{C}}\right)$ so that $\phi_{\gamma-\gamma(\beta)}^{\gamma} \pi_{2} \neq 0$.

5.7. LemMa. Let $\pi_{1}$ be a finite-dimensional irreducible representation of $G, \pi_{2}$ an infinite-dimensional irreducible representation such that

$$
\operatorname{Ext}_{U(\mathrm{~g})}^{1}\left(\pi_{1}, \pi_{2}\right) \neq 0 \text {. }
$$

Suppose $\beta \in \Sigma^{+}\left(g_{\mathbf{C}}, \mathfrak{h}_{\mathbf{C}}\right)$ is a simple root so that $\phi_{\gamma-\gamma(\beta)}^{\gamma} \pi_{2} \neq 0$. Then $\phi_{\gamma-\delta(\beta)}^{\gamma} \pi_{2}$ is a Langlands subquotient of exactly one of the direct summands of $\phi_{\gamma-\gamma(\beta)}^{\gamma} U\left(\pi_{1}\right)$.

Proof. Let $\pi$ be a nontrivial extension of $\pi_{1}$ by $\pi_{2}$. By Lemma 5.4 we have an exact sequence

$$
0 \rightarrow \pi \rightarrow U\left(\pi_{1}\right) \rightarrow U\left(\pi_{1}\right) / \pi \rightarrow 0
$$

and thus

$$
0 \rightarrow \phi_{\gamma-\gamma(\beta)}^{\gamma} \pi \rightarrow \phi_{\gamma-\gamma(\beta)}^{\gamma} U\left(\pi_{1}\right) \rightarrow \phi_{\gamma-\gamma(\beta)}^{\gamma}\left(U\left(\pi_{1}\right) / \pi\right) \rightarrow 0
$$


Since $\phi_{\gamma-\gamma(\beta)}^{\gamma} \pi=\phi_{\gamma-\gamma(\beta)}^{\gamma} \pi_{2} \neq 0, \phi_{\gamma-\gamma(\beta)}^{\gamma} \pi_{2}$ is a subrepresentation of $\phi_{\gamma-\gamma(\beta)}^{\gamma} U\left(\pi_{1}\right)$.

Let $C=T_{+} A$ be the Cartan subgroup of $G$ whose vector part is equal to the Iwasawa $A$. We may assume that $\mathfrak{h}_{\mathbf{C}}=\mathfrak{c}_{\mathbf{C}}$ and that $\Sigma^{+}\left(\mathfrak{g}_{\mathbf{C}}, \mathfrak{h}_{\mathbf{C}}\right)$ is compatible with $\Sigma_{P}^{+}$. Write $\gamma(\beta)=\left(\gamma_{1}(\beta), \gamma_{2}(\beta)\right)$, where $\gamma_{1}(\beta) \in t$, and $\gamma_{2}(\beta) \in a^{\prime}$.

If $\lambda \in \mathrm{t}^{\prime}$ write $\tau_{\lambda}$ for a finite-dimensional irreducible representation of $M$ whose highest weight vector transforms under $T_{+}$according to $\lambda$.

Assume $U\left(\pi_{1}\right)=U\left(P, \tau_{\lambda}, \chi\right)$. Then

$$
\phi_{\gamma-\gamma(\beta)}^{\gamma} U\left(P, \tau_{\lambda}, \chi\right)=U\left(P, \tau_{\lambda+\gamma_{1}(\beta)}, \chi+\gamma_{2}(\beta)\right)
$$

and $-\left(\chi+\gamma_{2}(\beta)\right)$ is dominant with respect to $\Sigma_{P}^{+}$, for the minimal parabolic subgroup $P$. Here $U\left(P, \tau_{\lambda+\gamma_{1}(\beta)}, \chi+\gamma_{2}(\beta)\right)$ is a direct sum of generalized principal series representations and each of these representations has exactly one subrepresentation, namely, its Langlands subquotient and all these Langlands subquotients are mutually inequivalent. Q.E.D.

5.8. THEOREM. Under the assumptions of Lemma 5.7

$$
\operatorname{dim} \operatorname{Ext}_{U(g)}^{1}\left(\pi_{1}, \pi_{2}\right)=1 .
$$

Proof. By Lemma 1.1 it suffices to show that there is only one equivalence class of extensions of $\pi_{1}$ by $\pi_{2}$.

Each extension $\pi$ of $\pi_{1}$ by $\pi_{2}$ is equivalent to a subrepresentation of $U\left(\pi_{1}\right)$. But by Lemma $5.7, \pi_{2}$ has multiplicity one in the Jordan-Hölder series of $U\left(\pi_{1}\right)$. Since $\operatorname{dim} \operatorname{Hom}_{U(g)}\left(\pi_{1}, U\left(\pi_{1}\right)\right)=1$, we have

$$
\operatorname{dim} \operatorname{Hom}_{U(g)}\left(\pi, U\left(\pi_{1}\right)\right)=1 \text {. Q.E.D. }
$$

5.9. Corollary. Let $\pi_{1}$ be a finite-dimensional irreducible representation of $G, \pi_{2}$ an infinite-dimensional irreducible representation such that

$$
\operatorname{Ext}_{U(\mathrm{~g})}^{1}\left(\pi_{1}, \pi_{2}\right) \neq 0 \text {. }
$$

Then

$$
\operatorname{dim} \operatorname{Ext}_{U(g)}^{1}\left(\pi_{1}, \pi_{2}\right)=1
$$

Proof. It suffices to show

$$
\operatorname{dim} \operatorname{Ext}_{U(\mathrm{~g})}^{1}\left(\pi_{1}, \pi_{2}\right)=\operatorname{dim} \operatorname{Ext}_{U(\mathrm{~g})}^{1}\left(\pi_{2}, \pi_{1}\right) .
$$

Let $\check{\pi}_{1}, \check{\pi}_{2}$ be the contragradient representations of $\pi_{1}$ and $\pi_{2}$. Then

$$
\operatorname{dim} \operatorname{Ext}_{U(\mathrm{~g})}^{1}\left(\pi_{1}, \pi_{2}\right)=\operatorname{dim} \operatorname{Ext}_{U(\mathrm{~g})}^{1}\left(\check{\pi}_{2}, \check{\pi}_{1}\right) \text {. }
$$

Since $\pi_{1}, \pi_{2}$ and $\tilde{\pi}_{1}, \tilde{\pi}_{2}$ are conjugate by an automorphism of $G[16,7.5]$

$$
\operatorname{dim} \operatorname{Ext}_{U(\mathfrak{g})}^{1}\left(\pi_{2}, \pi_{1}\right)=\operatorname{dim} \operatorname{Ext}_{U(\mathfrak{g})}^{1}\left(\tilde{\pi}_{2}, \tilde{\pi}_{1}\right) \text {. Q.E.D. }
$$

6. The main theorem. Assume $\pi_{1}$ is a finite-dimensional irreducible representation of $G, \pi_{2}$ an infinite-dimensional irreducible representation such that

$$
\operatorname{Ext}_{U(g)}^{1}\left(\pi_{1}, \pi_{2}\right) \neq 0 \text {. }
$$

In this section we estimate the dimension of $\operatorname{Ext}_{U(\mathrm{~g})}^{1}\left(\pi_{2}, \pi_{2}\right)$ and show that $\pi_{1}, \pi_{2}$ satisfy the assumptions (A)-(D) of $\S 2$. 
We start with some general considerations about principal series representations.

Let $\tau: P \rightarrow$ Aut $V$ be a representation of a parabolic subgroup $P=M A N$ such that $\tau(p n)=\tau(p)$ for $p \in P, n \in N$. Let $0=V_{0} \subset V_{1} \subset \cdots \subset V_{1}=V$ be a chain of closed $\tau$-invariant subspaces such that $V_{i} / V_{i-1}$ is the socle of $V / V_{i-1}$. Denote by $\tau^{i}$ the restriction of $\tau$ to $V_{i}$. Let $H$ be a closed $G$-invariant subspace of $\operatorname{ind}_{P}^{G} \tau$. Put $H_{i}=H \cap \operatorname{ind}_{P}^{G} \tau^{i}$ and let $m_{0}$ be the smallest index such that $H_{m_{0}}=H_{j}$ for all $j<m_{0}$.

6.1. Lemma. $H_{i} \subset H_{i+1}$ and $H_{i} \neq H_{i+1}$ for $i<m_{0}$.

Proof. Let $f \in \operatorname{ind}_{P}^{G} \tau$ and $f \in H_{i_{0}}$ but $f \notin H_{i_{0}-1}$. Then for $1 \in M A$

$$
f(1) \in V_{i_{0}}, \quad f(1) \notin V_{i_{0}-1} .
$$

But we can find a $Y \in U(\mathfrak{p})$, the enveloping algebra of $\mathfrak{p}$, such that

$$
\tau(Y) f(1) \in V_{i_{0}-1}, \quad \tau(y) f(1) \notin V_{i_{0}-2} .
$$

Put $I=\operatorname{ind}_{P}^{G} \tau$ and consider $I(Y) f$. Since $(I(Y) f)(1)=\tau(Y) f(1)$

$$
I(Y) f \in H_{i_{0}-1} \text { and } I(Y) f \notin H_{i_{0}-2} \text {. Q.E.D. }
$$

We also will make use of some results about intertwining operators [9], [15], which we will recall now.

Let $P=M A N$ be a parabolic subgroup of $G$. Write $\Sigma(g, a)$ for the roots of $a$ in g. We call $\Sigma^{+} \subset \Sigma(\mathfrak{g}, \mathfrak{a})$ a set of positive roots in $\Sigma(\mathfrak{g}, \mathfrak{a})$ if

(a) $\Sigma^{+} U-\Sigma^{+}=\Sigma(\mathfrak{g}, \mathfrak{a})$

(b) $\Sigma^{+} \cap-\Sigma^{+}=\varnothing$,

(c) if $\alpha, \beta \in \Sigma^{+}$and $\alpha+\beta \in \Sigma(g, a)$, then $\alpha+\beta \in \Sigma^{+}$.

Let $\Sigma^{+}, \bar{\Sigma}^{+}$be sets of positive roots. A sequence $\Sigma_{1}^{+}, \ldots, \Sigma_{n}^{+}$of sets of positive roots is a chain connecting $\Sigma^{+}$and $\bar{\Sigma}^{+}$if

(a) $\Sigma_{1}^{+}=\Sigma^{+}, \ldots, \Sigma_{n}^{+}=\bar{\Sigma}^{+}$,

(b) the span of $\Sigma_{i}^{+} \backslash\left(\Sigma_{i}^{+} \cap \Sigma_{i-1}^{+}\right)$is one dimensional.

The integer $n$ is called the length of the chain. For any two sets $\Sigma^{+}, \bar{\Sigma}^{+}$of positive roots there exists at least one chain connecting them. The length of the minimal chain is called the distance between $\Sigma^{+}$and $\bar{\Sigma}^{+}$.

For each set $\Sigma^{+}$of positive roots there exists a unique parabolic subgroup $Q=M A N_{Q}$ such that $\Sigma^{+}=\Sigma_{Q}^{+}$.

Let $U(P, \tau, \chi)$ be a generalized principal series representation. We assume that $\chi$ is nonsingular and strictly dominant with respect to $\Sigma_{P}^{+}$. Let $\bar{P}$ be the parabolic subgroup opposite to $P$.

6.2. ThEOREM [10], [15]. (a) There exists an intertwining operator

$$
I(P, \bar{P}, \tau, \chi): U(P, \tau, \chi) \rightarrow U(\bar{P}, \tau, \chi) .
$$

(b) If $Q=M A N_{Q}$ is another parabolic subgroup then there are intertwining operators

$$
\begin{aligned}
& I(P, Q, \tau, \chi): U(P, \tau, \chi) \rightarrow U(Q, \tau, \chi), \\
& I(Q, \bar{P}, \tau, \chi): U(Q, \tau, \chi) \rightarrow U(\bar{P}, \tau, \chi)
\end{aligned}
$$


such that

$$
I(P, \bar{P}, \tau, \chi)=I(Q, \bar{P}, \tau, \chi) I(P, Q, \tau, \chi) .
$$

(c) Let $\Sigma_{1}^{+}, \ldots, \Sigma_{r}^{+}$be a chain connecting $\Sigma_{Q}^{+}$and $\Sigma_{P}^{+}$and suppose $r$ is the distance between $\Sigma_{Q}^{+}$and $\Sigma_{P}^{+}$. Let $P_{i}$ be the parabolic subgroup with $\Sigma_{P_{i}}^{+}=\Sigma_{i}^{+}$. There exist intertwining operators

$$
I\left(P_{i}, P_{i+1}, \tau, \chi\right): U\left(P_{i}, \tau, \chi\right) \rightarrow U\left(P_{i+1}, \tau, \chi\right)
$$

such that

$$
I(Q, P, \tau, \chi)=I\left(P_{r-1}, P_{r}, \tau, \chi\right) \ldots I\left(P_{1}, P_{2}, \tau, \chi\right) .
$$

(d) $U(P, \tau, \chi)$ is cyclic, has a unique maximal closed invariant subspace and the image of $I(P, \bar{P}, \tau, \chi)$ is equal to the Langlands subquotient $U(\bar{P}, \tau, \chi)$ of $U(\bar{P}, \tau, \chi)$.

Let $I\left(P_{i}, P_{i+1}, \tau, \chi\right)$ be one of the operators of Theorem 6.2. Write $\check{P}_{i}=\check{M}_{i} \check{A}_{i} \check{N}_{i}$ for the smallest parabolic subgroup containing $P_{i}$ and $P_{i+1}$. Put $P_{i}=M_{i} A_{i} N_{i}$. Then

$$
\operatorname{ind}_{P_{i}}^{\dot{P}_{i}} \tau \otimes \chi=U\left(P_{i} \cap \check{M}_{i}, \tau, \chi_{\mid \dot{M}_{i} \cap A_{i} N_{i}}\right) \otimes \chi_{\mid A_{i} N_{i}}
$$

and $U\left(P_{i} \cap \check{M}_{i}, \tau, \chi_{\mid \check{M}_{i} \cap A_{i} N_{i}}\right)$ satisfies the assumptions of Theorem 6.2.

6.3. Proposition [15]. Suppose $I\left(P_{i}, P_{i+1}, \tau, \chi\right)$ is not an isomorphism. There exists a subrepresentation $V=V\left(\check{P}_{i}, \tau, \chi\right)$ of $U\left(P_{i} \cap M_{i}, \tau, \chi_{\mid M_{i} \cap A N}\right)$ such that

$$
\operatorname{ker} I\left(P_{i}, P_{i+1}, \tau, \chi\right)=\operatorname{ind}_{P_{i}}^{G} V \otimes \chi_{\mid A_{i} N_{i}} .
$$

$V\left(\check{P}_{i}, \tau, \chi\right)$ is the unique maximal nontrivial subrepresentation of

$$
U\left(P_{i} \cap M_{i}, \tau, \chi_{\mid M_{i} \cap A N}\right)
$$

6.4. Proposition. Let $P=M_{P} A_{P} N_{P}$ be a cuspidal parabolic subgroup of $G$ and $U(P, \tau, \chi)$ a generalized principal series representation with $\operatorname{Re} \chi$ strictly dominant with respect to $\Sigma_{P}$. Suppose $Q=M_{P} A_{P} N_{Q}$ is another parabolic subgroup. If

$$
\operatorname{Hom}_{U(\mathfrak{g})}(\overline{U(P, \tau, \chi)}, U(Q, \tau, \chi)) \neq 0
$$

then $U(P, \tau, \chi)$ and $U(Q, \tau, \chi)$ are isomorphic.

Proof. Let $\bar{P}$ be the parabolic subgroup opposite to $P$. Then by Proposition 6.3(d)

$$
I(P, \bar{P}, \tau, \chi) U(P, \tau, \chi)=\overline{U(\bar{P}, \tau, \chi)}
$$

Write $P=M A N$. Assume $\overline{U(P, \tau, \chi)}$ is a subrepresentation of $U(Q, \tau, \chi)$ for $Q=M A N_{Q}$. By Proposition 6.3(b),

$$
I(P, \bar{P}, \tau, \chi)=I(Q, \bar{P}, \tau, \chi) I(P, Q, \tau, \chi)
$$

and by Proposition 6.3(d),

$$
I(P, Q, \tau, \chi) U(P, \tau, \chi)=\overline{U(P, \tau, \chi)} .
$$

We will now show that $I(Q, \bar{P}, \tau, \chi)$ is an isomorphism. 
Let $r$ be the distance from $\Sigma_{Q}$ to $\Sigma_{P}$. By Proposition 6.3(c), there exists a sequence $P_{1}=Q, \ldots, P_{r}=P$ of parabolic subgroups such that

$$
I(Q, \bar{P}, \tau, \chi)=I\left(P_{r-1}, P_{i}, \tau, \chi\right) \ldots I\left(P_{1}, P_{2}, \tau, \chi\right) .
$$

So $I(Q, \bar{P}, \tau, \chi)$ is an isomorphism if each factor is an isomorphism.

Suppose $I(Q, P, \tau, \chi)$ is not an isomorphism and let $i_{0}$ be the first index $i$ for which $I\left(P_{i}, P_{i+1}, \tau, \chi\right)$ is not an isomorphism. Then $\overline{U(P, \tau, \chi)}$ is a subrepresentation of $U\left(P_{i}, \tau, \chi\right)$ and hence by Lemma 6.1 and Proposition $6.3 \overline{U(P, \tau, \chi)}$ is contained in the kernel of $I\left(P_{i}, P_{i+1}, \tau, \chi\right)$. Since $\overline{U(P, \tau, \chi)}$ occurs with multiplicity one in the Jordan-Hölder series of $U(P, \tau, \chi)$, we get a contradiction to (*). Q.E.D.

6.5. Proposition [14]. Let $P=M A N, Q=M A N_{Q}$ be minimal parabolic subgroups, $\chi \in a, \tau \in \hat{M}$.

(a) There exists an intertwining operator

$$
Q(P, Q, \tau, \chi): U(P, \tau, \chi) \rightarrow U(Q, \tau, \chi) .
$$

If $P, Q$ satisfy the assumptions of Theorem 6.2 , then $Q(P, Q, \tau, \chi)$ is a scalar multiple of $I(P, Q, \tau, \chi)$.

(b) Let $r$ be the distance between $\Sigma_{P}^{+}$and $\Sigma_{Q}^{+}$and let $\Sigma_{1}^{+}, \ldots, \Sigma_{r}^{+}$be a chain joining $\Sigma_{P}^{+}$and $\Sigma_{Q}^{+}$. Let $P_{i}$ be the parabolic subgroup with $\Sigma_{P_{i}}^{+}=\Sigma_{i}^{+}$. There exist intertwining operators

$$
\mathbb{Q}\left(P_{i}, P_{i+1}, \tau, \chi\right): U\left(P_{i}, \tau, \chi\right) \rightarrow U\left(P_{i+1}, \tau, \chi\right)
$$

such that

$$
\mathbb{Q}(P, Q, \tau, \chi)=c \mathbb{Q}\left(P_{r-1}, P_{r}, \tau, \chi\right) \ldots \mathscr{Q}\left(P_{1}, P_{2}, \tau, \chi\right)
$$

where $c=c(P, 0) \in \mathbf{C} \backslash 0$.

6.6. Proposition. Let $P=M A N$ be a minimal parabolic subgroup and $\chi \in a^{\prime}$ with $\operatorname{Re} \chi$ dominant with respect to $\Sigma_{P}^{+}$. Let $\tau \in \hat{M}$. Then $\mathcal{Q}(P, \bar{P}, \tau, \chi) U(P, \tau, \chi)$ is a direct sum of the irreducible subrepresentations of $U(P, \tau, \chi)$.

Proof. This follows from [10] and the construction of the operators $\mathbb{Q}(P, \bar{P}, \tau, \chi)$.

6.7. Corollary. Let $P=$ MAN be a minimal parabolic subgroup, $\alpha$ a simple root in $\Sigma_{P}^{+}$so that $\theta \alpha=\alpha, \chi \in a^{\prime}$ so that $\operatorname{Re}\langle\chi, \alpha\rangle=0$ and $\operatorname{Re}\langle\chi, \beta\rangle>0$ for $\beta \in \Sigma_{P}^{+}, \beta \neq \alpha$, and let $\tau \in \hat{M}$. Suppose $Q=M A N_{Q}$ is another parabolic subgroup, and $\pi_{0} \in \overline{U(P, \tau, \chi)}$. If

$$
\operatorname{Hom}_{U(\mathfrak{g})}\left(\pi_{0}, U(Q, \tau, \chi)\right) \neq 0
$$

then $U(P, \tau, \chi)$ and $U(Q, \tau, \chi)$ are isomorphic.

Proof. If $U(P, \tau, \chi)$ is indecomposable then $\pi_{0}=\overline{U(P, \tau, \chi)}$ and therefore we can use the same argument as in Proposition 6.4.

Suppose now $U(P, \tau, \chi)$ is not indecomposable. Then

$$
U(P, \tau, \chi)=U\left(P^{1}, \tau^{1}, \chi^{1}\right) \oplus U\left(P^{1}, \tau^{2}, \chi^{1}\right)
$$

where $P^{1}=M^{1} A^{1} N^{1}$ with $M^{1}=\tilde{M} \times \operatorname{Sl}(2, \mathbf{R})$ where $\tilde{M} \subset M$. Each summand is indecomposable. 
We denote by $\tau^{+}, \tau^{-}$the two composition factors of the reducible principal series representation of $\operatorname{SI}(2, \mathbf{R})$. Then $\tau^{1}=\tau^{+} \otimes \sigma, \tau^{2}=\tau^{-} \otimes \sigma$ with $\sigma \in \hat{\tilde{M}}$.

Let $T$ be the outer automorphism of $\operatorname{Sl}(2, \mathbf{R})$. We extend $T$ to an automorphism of $M^{1} A^{1}$. Thus

$$
\left(\tau^{+} \otimes \sigma \otimes \chi^{1}\right)^{T}=\tau^{-} \otimes \sigma \otimes \chi^{1}, \quad \text { and }\left(\tau^{-} \otimes \sigma \otimes \chi^{1}\right)^{T}=\tau^{+} \otimes \sigma \otimes \chi^{1} .
$$

So if $Q^{1}=M^{1} A^{1} N_{Q}$ is a parabolic subgroup, then

$$
U\left(Q^{1}, \tau^{i}, \chi^{1}\right)=U\left(Q^{1},\left(\tau^{j}\right)^{T}, \chi^{1}\right), \quad i \neq j, i, j \in\{1,2\} .
$$

So in particular

$$
\operatorname{Hom}_{U(g)}\left(\overline{U\left(P^{1}, \tau^{1}, \chi^{1}\right)}, U\left(Q^{1}, \tau^{1}, \chi^{1}\right)\right) \neq 0
$$

iff

$$
\operatorname{Hom}_{U(\mathrm{~g})}\left(\overline{U\left(P^{1}, \tau^{2}, \chi^{1}\right)}, U\left(Q^{1}, \tau^{2}, \chi^{1}\right)\right) \neq 0
$$

So we can by Proposition 6.6 use the same argument as in Propostion 6.4.

6.8. Proposition. Let $P=M A N$ be a minimal parabolic subgroup, $\chi \in \mathfrak{a}^{\prime}$, $\tau \in \hat{M}$. Suppose there exists a parabolic subgroup $P_{1} \supset P$ such that $\operatorname{ind}_{P}^{P_{1}} \tau \otimes \chi$ has a finite-dimensional subrepresentation $W=W\left(P_{1}, \tau, \chi\right)$. Let $Q=M A N_{Q}$ be a parabolic subgroup such that $P_{1}$ is the smallest parabolic subgroup containing $P$ and $Q$. Then

$$
\mathcal{Q}(Q, P, \tau, \chi) U(Q, \tau, \chi)=\operatorname{ind}_{P_{1}}^{G} W
$$

Proof. This follows from an argument similar to those of $\$ 3$ in [15]. The details are left to the reader. Q.E.D.

6.9. Corollary. Assume in addition that $\operatorname{Re} \chi$ is dominant with respect to $\Sigma_{P}^{+}$. The representation $\overline{U(P, \tau, \chi)}$ is isomorphic to a subrepresentation of $\operatorname{ind}_{P_{1}}^{G} W$.

Proof. This follows from Propositions 6.5 and 6.6. Q.E.D.

We now return to the problem of estimating $\operatorname{dim} \operatorname{Ext}_{U(\mathfrak{g})}^{1}\left(\pi_{2}, \pi_{2}\right)$.

6.10. Lemma. Suppose $\phi_{\gamma-\gamma(\beta)}^{\gamma} \pi_{2} \neq 0$ for a simple root $\beta$. Then $\operatorname{dim} \operatorname{Ext}_{U(\mathrm{~g})}^{1}\left(\pi_{2}, \pi_{2}\right)$ $\leqslant \operatorname{dim} \operatorname{Ext}_{U(\mathrm{~g})}^{1}\left(\phi_{\gamma-\gamma(\beta)}^{\gamma} \pi_{2}, \phi_{\gamma-\gamma(\beta)}^{\gamma} \pi_{2}\right)$.

Proof. Let $\pi$ be a nontrivial two-fold self-extension of $\pi_{2}$. Since $\phi_{\gamma-\gamma(\beta)}^{\gamma}$ is an exact functor, $\phi_{\gamma-\gamma(\beta)}^{\gamma} \pi$ is a two-fold self-extension of $\phi_{\gamma-\gamma(\beta)^{\gamma}}^{\gamma}$. It is nontrivial since

$$
\operatorname{dim} \operatorname{Hom}_{U(g)}\left(\phi_{\gamma-\gamma(\beta)}^{\gamma} \pi, \phi_{\gamma-\gamma(\beta)}^{\gamma} \pi_{2}\right)=\operatorname{dim} \operatorname{Hom}_{U(g)}\left(\pi, \psi_{\gamma-\gamma(\beta)}^{\gamma} \phi_{\gamma-\gamma(\beta)}^{\gamma} \pi_{2}\right)=1
$$

by [15], [17]. Q.E.D.

6.11. Proposition. Assume $\pi_{1}, \pi_{2}$ satisfy the assumptions of Lemma 5.6 and suppose $\phi_{\gamma-\gamma(\beta)}^{\gamma} \pi_{2} \neq 0$ for a simple root $\beta$. There exists (up to equivalence) exactly one principal series representation $U$ such that

$$
\operatorname{Hom}_{U(\mathrm{~g})}\left(\phi_{\gamma-\gamma(\beta)}^{\gamma} \pi_{2}, U\right) \neq 0 .
$$


Proof. We first show that there are at most $|W(\mathfrak{g}, \mathfrak{a})|$ inequivalent principal series representations which contain $\phi_{\gamma-\gamma(\beta)}^{\gamma} \pi_{2}$ as a subquotient.

We call $\chi$ the continuous parameter of the principal series representation $U(P, \tau, \chi)$ and write $\chi\left(\pi_{1}\right)$ for the continuous parameter of the principal series representation with Langlands subquotient $\pi_{1}$.

Let $\mathfrak{h}=\mathfrak{t} \oplus \mathfrak{a}$ be a maximally split Cartan subalgebra of $\mathfrak{g}$. For $\mu \in \mathfrak{h}^{\prime}$, put $\mu=\left(\mu_{1}, \mu_{2}\right)$ with $\mu_{1} \in \mathfrak{t}^{\prime}, \mu_{2} \in \mathfrak{a}^{\prime}$. Choose a set $\Sigma_{\mathfrak{a}}^{+}=\Sigma^{+}(\mathfrak{g}, \mathfrak{a})$ of positive roots so that $\chi\left(\pi_{1}\right)$ is strictly dominant with respect to $-\Sigma_{a}^{+}$. Let $\Sigma^{+}=\Sigma^{+}\left(g_{\mathbf{C}}, \mathfrak{h}_{\mathbf{C}}\right)$ be a set of positive roots compatible with the choice of $\Sigma_{a}^{+}$.

By Proposition 5.5 we may assume that $\pi_{1}$ is the trivial representation, i.e. that $\chi\left(\pi_{i}\right)=\rho_{P}, \gamma=\rho$ and $\gamma(\beta)=\delta(\beta)$.

Note first that $\beta$ is not an imaginary root since in that case $\beta$ is compact and hence by [15]

$$
\phi_{\rho-\delta(\beta)}^{\rho} U\left(\pi_{1}\right)=0 \text { and } \phi_{\rho-\delta(\beta)}^{\rho} \pi_{2}=0 .
$$

Now assume $\beta$ is a real or complex root. Then $\rho-\delta(\beta)=\left(\rho_{c}-\rho^{0}, \rho^{2}\right)$, where $\rho_{c}$ is half the sum of the compact roots and $\left(\rho^{0}, \alpha\right)=0$ for all compact roots $\alpha$.

By Theorem 7.3.2 in the Erratum Appendix to Chapter 7 of [1] $\phi_{\rho-\delta(\beta)}^{\rho} \pi_{2}$ cannot be a composition factor of a principal series representation $U(P, \tau, \chi)$ with infinitesimal character $\rho-\delta(\beta)$ if $\left.\left\langle\rho^{2}, \rho^{2}\right\rangle\right\rangle\langle\chi, \chi\rangle$. But the choice of $\Sigma^{+}(\mathfrak{g}, \mathfrak{h})$ implies that if $\mu=\left(\mu^{1}, \mu^{2}\right) \in \mathfrak{h}^{\prime}$ is conjugate under $W\left(\mathfrak{g}_{\mathbf{C}}, \mathfrak{h}_{\mathbf{C}}\right)$ to $\rho-\delta(\beta)$, then $\left\langle\rho^{2}, \rho^{2}\right\rangle$ $\leqslant\left\langle\mu^{2}, \mu^{2}\right\rangle$. Hence $\phi_{\rho-\delta(\beta)}^{\rho} \pi_{2}$ is a subrepresentation of a principal series representation $U(P, \tau, \chi)$ with $\langle\chi, \chi\rangle=\left\langle\rho^{2}, \rho^{2}\right\rangle$. So in particular $\chi$ is conjugate to $\rho^{2}$ under the Weyl group $W_{\mathrm{a}}$ of $\Sigma(\mathfrak{a}, \mathfrak{g})$.

Since $\phi_{\rho-\delta(\beta)}^{\rho} \pi_{2}$ is contained in the Langlands subquotient of $U(P, \tau, \mu)$ for some $\tau \in \hat{M}, \mu \in\left\{w \rho^{2}, w \in W_{\mathrm{a}}\right\}$, we have $\rho^{2} \in \xi^{0}\left(\phi_{\rho-\delta(\beta)}^{\rho} \pi_{2}\right)$. The only subquotients of principal series representations whose leading exponent is conjugate to $\rho^{2}$ are the Langlands subquotients. So there exists a unique $\tau_{0} \in \hat{M}$, so that $\phi_{\rho-\delta(\beta)}^{\rho} \pi_{2}$ is a subquotient of $U\left(P, \tau_{0}, \chi\right), \chi \in\left\{w \rho^{2}, w \in W_{\mathrm{a}}\right\}$.

Suppose $U\left(P, \tau_{0}, \chi_{0}\right)$ is a principal series representation so that $\operatorname{Re} \chi_{0}$ is dominant with respect to $-\Sigma_{P}^{+}$and

$$
\operatorname{Hom}_{U(\beta)}\left(\phi_{\rho-\delta(\beta)}^{\rho} \pi_{2}, U\left(P, \tau_{0}, \chi_{0}\right)\right) \neq 0 .
$$

To prove the proposition it suffices to show that if for some parabolic $Q$

$$
\operatorname{Hom}_{U(\mathrm{~g})}\left(\phi_{\rho-\delta(\beta)}^{\rho} \pi_{2}, U\left(Q, \tau_{0}, \chi_{0}\right)\right) \neq 0
$$

then $U\left(P, \tau_{0}, \chi_{0}\right) \cong U\left(Q, \tau_{0}, \chi_{0}\right)$. We first assume that $\beta$ is a complex root.

6.12. Lemma. Suppose $\beta$ is a complex root and $\rho-\delta(\beta)=\left(\rho_{c}-\rho^{0}, \rho^{2}\right)$. Then $\rho^{2}$ is strictly dominant with respect to $\Sigma_{P}^{+}$.

Thus $\phi_{\rho-\delta(\beta)}^{\rho} \pi_{2}$ is a Langlands subquotient of $U\left(P, \tau_{0}, \chi_{0}\right)$. So Proposition 6.4 implies the result.

Now assume $\beta$ is a real root. Then $\phi_{\rho-\delta(\beta)}^{\rho} \pi_{2} \in \overline{U\left(P, \tau_{0}, \chi_{0}\right)}$. So Corollary 6.7 and Proposition 6.4 imply the result. Q.E.D.

Proof of Lemma 6.12. The restriction $\delta^{2}(\beta)$ of $\delta(\beta)$ to a is contained in the closed dominant Weyl chamber $\mathcal{C}_{\mathbf{C}}^{+} \subset \mathfrak{h}$, since $-\theta(\alpha) \in \Sigma^{+}\left(\mathfrak{g}_{\mathbf{C}}, \mathfrak{h}_{\mathbf{C}}\right)$ for a complex 
simple root $\alpha$. It suffices to show that for each simple complex root $\alpha \in \Sigma^{+}\left(\mathfrak{g}_{\mathbf{C}}, \mathfrak{h}_{\mathbf{C}}\right)$

$$
\left(\rho_{P}-\delta^{2}(\beta), \alpha-\theta(\alpha)\right)>0 \text {. }
$$

But since $\alpha-\theta(\alpha) \neq \beta$

$$
\left(\rho_{P}-\delta^{2}(\beta), \alpha-\theta(\alpha)\right)=(\rho-\delta(\beta), \alpha-\theta(\alpha))>0 \text {. Q.E.D. }
$$

6.13. Lemma. Assume $\pi_{1}, \pi_{2}$ satisfy the assumptions of Corollary 5.9, and suppose $\phi_{\gamma-\gamma(\beta)}^{\gamma} \pi_{2} \neq 0$. The representation $\phi_{\gamma-\gamma(\beta)}^{\gamma} \pi_{2}$ is a subrepresentation of a degenerate series representation induced from a maximal parabolic subgroup.

Proof. Let $U\left(P_{0}, \tau_{0}, \chi_{0}\right)$ be a principal series representation so that

$$
\operatorname{Hom}_{U(g)}\left(\phi_{\gamma-\gamma(\beta)}^{\gamma} \pi_{2}, U\left(P_{0}, \tau_{0}, \chi_{0}\right)\right) \neq 0
$$

and $\chi_{0}$ dominant with respect to $-\Sigma_{P_{0}}^{+}$.

Suppose there exists a degenerate series representation

$$
D\left(P^{1}, \tau_{1}^{1}, \chi^{1}\right)=\operatorname{ind}_{P^{1}}^{G} \tau^{1} \otimes \chi^{1} \otimes \text { id }
$$

with $P^{1}$ maximal and $D\left(P^{1}, \tau^{1}, \chi^{1}\right)$ a subrepresentation of $U\left(P_{0}, \tau_{0}, \chi_{0}\right)$. Then $-\chi^{1}$ is dominant with respect to $\Sigma_{P^{1}}^{+}$and $D\left(P^{1}, \tau^{1}, \chi^{1}\right)$ is a subrepresentation of $U\left(Q, \tau_{0}, \chi_{0}\right)$, where $Q$ is a minimal parabolic in $P^{1}$ minimalizing the distance between $\Sigma_{P_{0}}^{+}$and $\Sigma_{Q}^{+}$.

By Corollary $6.9 \phi_{\gamma-\gamma(\beta)}^{\gamma} \pi_{2}$ is a composition factor of $D\left(P^{1}, \tau^{1}, \chi^{1}\right)$ and since it occurs with multiplicity one in $U\left(P_{0}, \tau_{0}, \chi_{0}\right)$ we have

$$
\operatorname{Hom}_{U(\mathrm{~g})}\left(\phi_{\gamma-\gamma(\beta)}^{\gamma} \pi_{2}, D\left(P^{1}, \tau^{1}, \chi^{1}\right)\right) \neq 0 .
$$

We now prove the existence of such a representation $D\left(P^{1}, \tau^{1}, \chi^{1}\right)$. Let $U(P, \tau, \chi)$ be the principal series representation with Langlands subquotient $\pi_{1}, \Sigma_{\mathrm{a}}^{+}$ and $\Sigma^{+}$the set of positive roots in $\Sigma(\mathrm{g}, \mathrm{a})$ and $\Sigma\left(\mathrm{g}_{\mathrm{C}}, \mathrm{a} \oplus \mathrm{t}\right)$ determined through $P=M A N$. Using induction by stages we can find for each maximal parabolic $P^{1}=M^{1} A^{1} N^{1} \supset P$ a degenerate series representation $D\left(P^{1}, \tau_{P}, \chi_{P}\right)$ with

$$
\operatorname{Hom}_{U(\mathrm{~g})}\left(D\left(P^{1}, \tau_{P}, \chi_{P}\right), U(P, \tau, \chi)\right) \neq 0 .
$$

By Proposition 5.5 we may assume that $\pi_{1}$ is the one-dimensional representation. So $\chi_{P}=-\rho_{P}$, and $\tau_{P}$ is the identity representation Id.

Let $\beta$ be a simple root in $\Sigma^{+}$. As for connected semisimple groups we define the functor $\phi$ in the case of a reductive group with finitely many connected components. Then by [15]

$$
\phi_{\rho-\delta(\beta)}^{\rho} D\left(P^{1}, \mathrm{Id},-\rho_{P}\right)=\operatorname{ind}_{P}^{G} \phi_{\rho-\delta(\beta)}^{\rho}\left(\mathrm{Id} \otimes-\rho_{P}\right) \otimes \mathrm{id}
$$

and

$$
\phi_{\rho-\delta(\beta)}^{\rho}\left(\mathrm{Id} \otimes-\rho_{P}\right)=0
$$

iff $\beta \in \Sigma\left(\mathfrak{n}^{1} \oplus \mathfrak{a}^{1}, \mathfrak{a} \oplus \mathfrak{t}\right)$.

So if $P_{\beta}=M_{\beta} A_{\beta} N_{\beta} \supset P$ is a maximal parabolic subgroup such that the restriction of $\beta$ to $a_{P}$ is nontrivial, then

$$
\phi_{\rho-\delta(\beta)}^{\rho} D\left(P_{\beta}, \mathrm{Id},-\rho_{P}\right) \neq 0 \text {. Q.E.D. }
$$


6.14. Corollary. Assume $\pi_{1}, \pi_{2}$ satisfy the assumptions of Corollary 5.9 and suppose $\phi_{\gamma-\gamma(\beta)}^{\gamma} \pi_{2} \neq 0$. Let $D\left(P^{1}, \tau^{1}, \chi^{1}\right)$ be the degenerate series representation constructed in Lemma 6.13. Each embedding of $\phi_{\gamma-\gamma(\beta)}^{\gamma} \pi_{2}$ into a principal series representation $U(P, \tau, \chi)$ factors through an embedding of $D\left(P^{1}, \tau^{1}, \chi^{1}\right)$ into $U(P, \tau, \chi)$.

Proof. By the proof of Lemmas 6.13 and $6.10, D\left(P^{1}, \tau^{1}, \chi^{1}\right)$ is a subrepresentation of $U(P, \tau, \chi)$. Q.E.D.

6.15. Proposition. Assume $\pi_{1}, \pi_{2}$ satisfy the assumptions of Corollary 5.9 and suppose $\phi_{\gamma-\gamma(\beta)}^{\gamma} \pi_{2} \neq 0$. Let $D\left(P^{1}, \tau^{1}, \chi^{1}\right)$ be the degenerate series representation constructed in Lemma 6.13. Suppose $\pi_{\beta}^{2}$ is a two-fold self-extension of $\phi_{\gamma-\gamma(\beta)}^{\gamma} \pi_{2}$. Then there exists a two-fold self-extension $\left(\chi^{1}\right)^{2}$ of $\chi^{1}$ such that $\pi_{\beta}^{2}$ is equivalent to $a$ subrepresentation of ind $_{P^{1}}^{G} \tau^{1} \otimes\left(\chi^{1}\right)^{2} \otimes$ id.

Proof. Let $U(P, \tau, \chi)$ be the principal series representation such that

$$
\operatorname{Hom}_{U(\mathrm{~g})}\left(\phi_{\gamma-\gamma(\beta)}^{\gamma} \pi_{2}, U(P, \tau, \chi)\right) \neq 0
$$

and $\operatorname{Re} \chi$ negative with respect to $\Sigma_{P}$. By Lemmas 4.4 and 6.10 there exists a two-fold self-extension $\chi^{2}$ of $\chi$ such that $\pi_{\beta}^{2}$ is equivalent to a subrepresentation of ind $_{P}^{G} \tau \otimes \chi^{2} \otimes$ id.

Write $P^{1}=M^{1} A^{1} N^{1}, P=M A N$ and $\check{P}=P \cap M^{1} A^{1}$. By Corollary 6.14 we have an exact sequence

$$
0 \rightarrow \tau^{1} \chi^{1} \rightarrow \operatorname{ind}_{\ddot{P}}^{M^{1} A^{1}} \tau \otimes \chi^{2} \rightarrow\left(\operatorname{ind}_{\vec{P}}^{M^{1} A^{1}} \tau \otimes \chi^{2}\right) /\left(\tau^{1} \otimes \chi^{1}\right) \rightarrow 0 .
$$

Let $\left(\left(\text { ind }_{\vec{P}}^{M^{\prime} A^{\prime}} \tau \otimes \chi^{2}\right) /\left(\tau^{1} \otimes \chi^{2}\right)\right)_{s}$ be the socle of $\left(\right.$ ind $\left._{\vec{P}}^{M^{\prime} A^{\prime}} \tau \otimes \chi^{2}\right) /\left(\tau^{1} \otimes \chi^{2}\right)$. Since

$$
\operatorname{Hom}_{U(g)}\left(\phi_{\gamma-\gamma(\beta)}^{\gamma} \pi_{2}, \operatorname{ind}_{P^{1}}^{G}\left(\left(\operatorname{ind}_{\vec{P}}^{M^{1} A^{1}} \tau \otimes \chi^{2}\right) /\left(\tau^{1} \otimes \chi^{1}\right)\right)_{s} \otimes \text { id }\right) \neq 0
$$

and since $\phi_{\gamma-\gamma(\beta)}^{\gamma} \pi_{2}$ occurs with multiplicity one in the composition series of $U(P, \tau, \chi)$, Lemma 6.1 implies

$$
\operatorname{Hom}_{U\left(n \oplus a^{1}\right)}\left(\tau^{1} \otimes \chi^{1},\left(\operatorname{ind}_{\vec{P}}^{M^{1} A^{1}} \tau \otimes \chi^{2} / \tau^{1} \otimes \chi^{1}\right)_{s}\right) \neq 0
$$

So there exists a two-fold self-extension $\left(\tau^{1} \otimes \chi^{1}\right)^{2}$ so that the sequence

$$
0 \rightarrow\left(\tau^{1} \otimes \chi^{1}\right)^{2} \rightarrow \operatorname{ind}_{\vec{P}}^{M^{1} A^{1}} \tau \otimes \chi^{2} \rightarrow\left(\operatorname{ind}_{\ddot{P}}^{M^{1} A^{1}} \tau \otimes \chi^{2}\right) /\left(\tau^{1} \otimes \chi^{1}\right)^{2} \rightarrow 0
$$

is exact. Since $\tau^{1}$ is finite dimensional, by Lemma $3.1\left(\tau^{1} \otimes \chi^{1}\right)^{2}$ is isomorphic to a representation $\tau^{1} \otimes\left(\chi^{1}\right)^{2}$ where $\left(\chi^{1}\right)^{2}$ is a two-fold self-extension of $\chi^{1}$. Using that induction is an exact functor, we get an exact sequence

$$
\begin{aligned}
0 & \rightarrow \operatorname{ind}_{P}^{G} \tau^{1} \otimes\left(\chi^{1}\right)^{2} \otimes \mathrm{id} \rightarrow \operatorname{ind}_{P}^{G} \tau \otimes \chi^{2} \otimes \mathrm{id} \\
& \rightarrow \operatorname{ind}_{P}^{G}\left(\operatorname{ind}_{P}^{M^{\prime} A^{1}} \tau \otimes(\chi)^{2}\right) /\left(\tau^{1} \otimes\left(\chi^{1}\right)^{2}\right) \otimes \mathrm{id} \rightarrow 0 .
\end{aligned}
$$

Since all composition factors isomorphic to $\phi_{\gamma-\gamma(\beta)}^{\gamma} \pi_{2}$ are composition factors of ind $_{P^{1}}^{G} \tau^{1} \otimes\left(\chi^{1}\right)^{2} \otimes$ id, the proposition follows. Q.E.D.

6.16. TheOREM. Assume $\pi_{1}$ is a finite-dimensional irreducible representation of $G$, $\pi_{2}$ an infinite-dimensional irreducible representation such that

$$
\operatorname{Ext}_{U(\mathrm{~g})}^{1}\left(\pi_{1}, \pi_{2}\right) \neq 0 \text {. }
$$

Then $\operatorname{dim} \operatorname{Ext}_{U(\mathrm{~g})}^{1}\left(\pi_{2}, \pi_{2}\right) \leqslant 1$. 
Proof. By Lemma 6.10 it suffices to estimate the dimension of

$$
\operatorname{Ext}_{U(g)}^{1}\left(\phi_{\gamma-\gamma(\beta)}^{\gamma} \pi_{2}, \phi_{\gamma-\gamma(\beta)}^{\gamma} \pi_{2}\right)
$$

for a simple root $\beta$ with $\phi_{\gamma-\gamma(\beta)}^{\gamma} \pi_{2} \neq 0$. By Proposition 6.15 there exists a degenerate series representation $D\left(P^{1}, \tau^{1}, \chi^{1}\right)$ induced from a maximal parabolic subgroup $P^{1}$ such that

$$
\operatorname{Hom}_{U(g)}\left(\phi_{\gamma-\gamma(\beta)}^{\gamma} \pi_{2}, D\left(P^{1}, \tau^{1}, \chi^{1}\right)\right) \neq 0
$$

and such that each two-fold self-extension of $\phi_{\gamma}^{\gamma}-\gamma(\beta) \pi_{2}$ is isomorphic to a subrepresentation of $\operatorname{ind}_{P^{1}}^{G} \tau^{1} \otimes\left(\chi^{1}\right)^{2} \otimes$ id for a two-fold self-extension $\left(\chi^{1}\right)^{2}$ of $\chi^{1}$. Hence there are at most two equivalence classes of self-extensions of $\phi_{\gamma-\gamma(\beta)}^{\gamma} \pi_{2}$. Q.E.D.

6.17. TheOREM. Assume $\pi_{1}$ is a finite-dimensional irreducible representation, $\pi_{2}$ an infinite dimensional irreducible representation such that

$$
\operatorname{Ext}_{U(g)}^{1}\left(\pi_{1}, \pi_{2}\right) \neq 0 .
$$

Then $H\left(\pi_{1}, \pi_{2}\right)$ is isomorphic to a subcategory of $\mathfrak{X}$.

Proof. Since $\pi_{1}$ is an irreducible finite-dimensional representation

$$
\operatorname{Ext}_{U(g)}^{1}\left(\pi_{1}, \pi_{1}\right)=0 .
$$

So $\pi_{1}, \pi_{2}$ satisfy conditions (A)-(D) of $\S 2$.

If $\pi_{1}$ is one dimensional then condition (E) is satisfied. Since the trivial $K$-type has multiplicity one in a principal series representation and since each irreducible representation of $G$ contains at least one $K$-type with multiplicity one [17], the result follows by applying Theorems 5.6 and 3.17. Q.E.D.

\section{BIBLIOGRAPHY}

1. A. Borel and N. Wallach, Seminar on the cohomology of discrete subgroups of semisimple groups, Institute for Advanced Study, Princeton, N. J., 1976-1977.

2. W. Casselman, The differential equations satisfied by matrix coefficients, manuscript, 1975.

3. V. Dlab and C. Ringel, Indecomposable representations of groups and algebras, Mem. Amer. Math. Soc. No. 173 (1976).

4. I. M. Gelfand, The cohomology of infinite dimensional Lie algebras; some questions of integral geometry, Proc. Internat. Congr. Math. (Nice, 1970), Gauthier-Villars, Paris, 1971, pp. 95-111.

5. I. M. Gelfand, M. I. Graev and V. A. Ponomarev, The classification of the linear representations of the group SL(2, C), Soviet Math. Dokl. 11 (1970), 1319-1323.

6. I. M. Gelfand and V. A. Ponomarev, The category of Harish-Chandra modules over the Lie algebra of the Lorentz group, Soviet. Math. Dokl. 8 (1967), 1065-1068.

7. Classification of indecomposable infinitesimal representations of the Lorentz group, Soviet. Math. Dokl. 8 (1967), 1114-1117.

8. Harish-Chandra, Representations of semisimple Lie groups. III, Trans. Amer. Math. Soc. 76 (1954), $26-65$.

9. A. W. Knapp and E. M. Stein, Singular integrals and the principal series. IV, Proc. Nat. Acad. Sci. U.S.A. 72 (1975), 2459-2461.

10. R. P. Langlands, On the classification of irreducible representations of real algebraic groups, Institute for Advanced Study, Princeton, N. J., mimeographed notes, 1973.

11. D. Milicic, Asymptotic behaviour of matrix coefficients of the discrete series, Duke Math. J. 44 (1977), 59-88.

12. __ Jacquet modules for real groups and Langlands classification of representations, Lecture in Oberwolfach, June 1977.

13. V. Dlab and P. Gabriel, Representations of algebras, Lecture Notes in Math., vol. 488, SpringerVerlag, Berlin and New York, 1975. 
14. G. Schiffman, Integrales d'enterlacement et fonctions de Whittaker, Bull. Soc. Math. France 99 (1971), 3-72.

15. B. Speh and D. A. Vogan, Reducibility of generalized principal series representations, Acta Math. (to appear).

16. D. A. Vogan, Irreducible characters of semisimple Lie groups. 1, Duke Math. J. 46 (1979), 61-108.

17. _Lie algebra cohomology and the representations of semisimple Lie groups, Thesis, M.I.T., Cambridge, Mass., 1976.

18. G. Zuckerman, Tensor products of finite and infinite dimensional representations of semisimple Lie groups, Ann. of Math. (2) 106 (1977), 295-308.

Department of Mathematics, Gesamthochschule 1 Wuppertal, Fachbereich Mathematik, 56 WuPPERTAL, West Germany

Current address: Department of Mathematics, Cornell University, Ithaca, New York 14850 